\title{
Numerical twist-even SU(1,1)-singlet solutions in open string field theory around the identity-based solution
}

\author{
Isao Kishimoto $^{a}$ and Tomohiko Takahashi ${ }^{b}$ \\ ${ }^{a}$ Center for Liberal Arts and Sciences, Sanyo-Onoda City University, \\ Daigakudori 1-1-1, Sanyo-Onoda Yamaguchi 756-0884, Japan \\ ${ }^{b}$ Department of Physics, Nara Women's University, \\ Nara 630-8506, Japan \\ E-mail: ikishimo@rs.socu.ac.jp, tomo@asuka.phys.nara-wu.ac.jp
}

ABSTRACT: Using the level truncation method, we construct numerical solutions, which are twist even and SU(1,1) singlet, in the theory around the Takahashi-Tanimoto identitybased solution (TT solution) with a real parameter $a$ in the framework of bosonic open string field theory. We find solutions corresponding to "double brane" and "ghost brane" solutions which were constructed by Kudrna and Schnabl in the conventional theory around the perturbative vacuum. Our solutions show somewhat similar $a$-dependence to tachyon vacuum and single brane solutions, which we found in the earlier works. In this sense, we might be able to expect that they are consistent with the conventional interpretation of $a$-dependence of the TT solution. We observe that numerical complex solutions at low levels become real ones at higher levels for some region of the parameter $a$. However, these real solutions do not so improve interpretation for double brane.

Keywords: Bosonic Strings, D-branes, String Field Theory

ARXIV EPRINT: 2011.08378 


\section{Contents}

1 Introduction 1

2 Procedure for constructing numerical solutions 2

3 "Double brane" solution 5

3.1 Energy 6

$\begin{array}{ll}3.2 & \text { Gauge invariant observable }\end{array}$

$3.3\left|\boldsymbol{\Delta}_{S}\right|$ and reality 9

4 "Ghost brane" solution $\quad 10$

$\begin{array}{lll}4.1 & \text { Energy } & 11\end{array}$

$\begin{array}{ll}4.2 & \text { Gauge invariant observable } \\ \end{array}$

$\begin{array}{lll}4.3 & \left|\Delta_{S}\right| \text { and reality } & 13\end{array}$

5 Concluding remarks $\quad 15$

$\begin{array}{ll}\text { A Some numerical data for solutions } & 17\end{array}$

$\begin{array}{lll}\text { A.1 Numerical data for solutions at } a=-1 / 2 & 17\end{array}$

$\begin{array}{lll}\text { A.2 Coefficients of lowest level states for the solutions } & 18\end{array}$

A.3 Evaluation of quadratic identities 21

\section{Introduction}

In bosonic open string field theory, there is a well-known numerical solution: the tachyon vacuum solution in Siegel gauge. It was found by Sen and Zwiebach [1] using the level truncation method and then higher level calculations were performed for the solution [25]. It is known that efficient and consistent truncation for it can be obtained by restricting the space of string fields to that spanned by twist even and $\mathrm{SU}(1,1)$ singlet states. With the same restriction, but relaxing the reality condition, Kudrna and Schnabl constructed two interesting solutions [5], which might be interpreted as double brane and ghost brane, respectively.

Numerical solutions mentioned above can be constructed using Newton's method by choosing appropriate initial configurations so that iterative calculations converge. The tachyon vacuum solution can be uniquely obtained from a real solution at the level 0 , namely, the lowest truncation level. The "double brane" and "ghost brane" solutions can be obtained from one of complex solutions, which do not satisfy the reality condition for string fields, at the truncation level 2 and 4, respectively.

On the other hand, the Takahashi-Tanimoto (TT) solution [6] is based on the identity string field and therefore direct evaluation of its energy was difficult. Alternatively, in the theory around the TT solution, the BRST cohomology was studied [7] and numerical 
solutions in Siegel gauge were investigated [8-10]. The TT solution has a real parameter $a$ such as $a \geq-1 / 2$ and it is expected that it represents the tachyon vacuum at $a=-1 / 2$ and is pure gauge for $a>-1 / 2$. It was supported by analysis of cohomology in [7] and evaluation of energy of the tachyon vacuum solution in Siegel gauge [8] in the theory around the TT solution $\left(\Psi_{a}^{\mathrm{TT}}\right)$. Furthermore, numerical solution for unstable vacuum, which corresponds to the perturbative vacuum or single brane, was found in [9] in the theory around $\Psi_{a}^{\mathrm{TT}}$ for $a \simeq-1 / 2 .^{1}$

In this paper, motivated by "double brane" and "ghost brane" solutions found in [5], we construct numerical twist-even SU(1,1)-singlet solutions, which correspond to them, in the theory around $\Psi_{a}^{\mathrm{TT}}$ for $a \geq-1 / 2$. They show somewhat similar $a$-dependence to the tachyon vacuum and single brane solutions. Namely, with increasing level, energy of them seems to approach a constant $E$ for $a>-1 / 2$ and another value $E^{\prime}$ for $a \simeq-1 / 2$ $\left(E^{\prime}>E\right)$. However, the numerical behaviors of them are not so clear. Actually, the values of energy are complex in general because these numerical solutions are obtained from one of complex solutions in the truncation level 2 and 4 . Roughly, the imaginary part of the energy for them seems to approach zero with increasing level. Particularly, we find that these solutions in the theory around $\Psi_{a}^{\mathrm{TT}}$ for some region of $a$ satisfy the reality condition at higher levels although they start from complex solutions at the level 2 or 4 .

Here, we have performed numerical calculations up to the truncation level 22, and the values of the energy and the gauge invariant observable ${ }^{2}$ for "double brane" and "ghost brane" solutions might (not) represent the number of branes literally. Further investigation is necessary for a definite interpretation.

This paper is organized as follows. In section 2, we will explain our strategy of numerical calculations and conventions briefly. In section 3 and section 4 , we will show our numerical results: plots of energy, gauge invariant observables and so on, for "double brane" solution and "ghost brane" solution, respectively. In section 5, we will give some remarks on our calculations. Moreover, in appendix A, we will give some explicit numerical data for the solutions at $a=-1 / 2$ including evaluations of quadratic identities.

\section{Procedure for constructing numerical solutions}

In this section, we briefly explain procedure to construct numerical solutions and we define quantities to evaluate for the solutions. Further technical details can be found in [5].

The TT solution $\Psi_{a}^{\mathrm{TT}}[6]$, with a real parameter $a$ such as $a \geq-1 / 2$, is one of identitybased solutions to the equation of motion $Q_{\mathrm{B}} \Psi+\Psi * \Psi=0$ in open string field theory, whose action is $S[\Psi]=-\frac{1}{g^{2}}\left(\frac{1}{2}\left\langle\Psi, Q_{\mathrm{B}} \Psi\right\rangle+\frac{1}{3}\langle\Psi, \Psi * \Psi\rangle\right)$, where $Q_{\mathrm{B}}$ is the conventional BRST operator. Around the TT solution $\Psi_{a}^{\mathrm{TT}}$, we define the action $S_{a}[\Phi] \mathrm{as}^{3}$

$$
S_{a}[\Phi]=S\left[\Psi_{a}^{\mathrm{TT}}+\Phi\right]-S\left[\Psi_{a}^{\mathrm{TT}}\right]=-\frac{1}{g^{2}}\left(\frac{1}{2}\left\langle\Phi, Q^{\prime} \Phi\right\rangle+\frac{1}{3}\langle\Phi, \Phi * \Phi\rangle\right),
$$

\footnotetext{
${ }^{1}$ The energy of $\Psi_{a}^{\mathrm{TT}}$ is calculated analytically in $[11,12]$ and it is confirmed that the TT solution is the tachyon vacuum at $a=-1 / 2$ and is pure gauge for $a>-1 / 2$.

${ }^{2}$ It is also called gauge invariant overlap or Ellwood invariant in the literatures.

${ }^{3}$ We take the case of $l=1$ for the TT solution, where an integer $l$ was introduced in [7]. Similar computations can be performed for $l=2,3,4, \cdots$, but $l=1$ is the most fundamental in the context of the level truncation in the sense that mixing of the levels in the kinetic term is minimum in Siegel gauge.
} 


$$
\begin{aligned}
Q^{\prime}= & (1+a) Q_{\mathrm{B}}+\frac{a}{2}\left(Q_{2}+Q_{-2}\right)+4 a Z(a) c_{0}-2 a Z(a)^{2}\left(c_{2}+c_{-2}\right) \\
& +2 a\left(1-Z(a)^{2}\right) \sum_{n=2}^{\infty}(-Z(a))^{n-1}\left(c_{2 n}+c_{-2 n}\right), \\
Z(a)= & \frac{1+a-\sqrt{1+2 a}}{a},
\end{aligned}
$$

where $Q_{n}$ is a mode of the BRST current. In the case $a=0, \Psi_{a}^{\mathrm{TT}}$ becomes zero, namely $\Psi_{a=0}^{\mathrm{TT}}=0$, and hence $\left.Q^{\prime}\right|_{a=0}=Q_{\mathrm{B}}$. The equation of motion of (2.1) is

$$
Q^{\prime} \Phi+\Phi * \Phi=0
$$

and it is projected to

$$
\begin{aligned}
L(a) \Phi+b_{0}(\Phi * \Phi)= & 0, \\
L(a)= & (1+a)\left(L_{0}^{\mathrm{mat}}+L_{0}^{\text {gh }}-1\right)+\frac{a}{2}\left(L_{2}^{\mathrm{mat}}+L_{2}^{\mathrm{gh}}+L_{-2}^{\mathrm{mat}}+L_{-2}^{\mathrm{gh}}\right) \\
& +4(1+a-\sqrt{1+2 a}),
\end{aligned}
$$

using the Siegel gauge condition: $b_{0} \Phi=0$. Here, $L_{n}^{\text {mat }}$ is the Virasoro generator in the matter sector with the central charge 26 and $L_{n}^{\text {gh }}$ is a twisted Virasoro generator in the ghost sector with the central charge -2 , which is given by

$$
L_{n}^{\text {gh }}=\sum_{m=-\infty}^{\infty}(n-m): b_{m} c_{n-m}:
$$

in terms of the $b c$-ghost modes. In order to solve a nonlinear equation (2.5), we use Newton's method as follows. Firstly, we take an initial string field $\Phi^{(0)}$, then we solve a linearized equation:

$$
L(a) \Phi^{(n+1)}+b_{0}\left(\Phi^{(n)} * \Phi^{(n+1)}\right)+b_{0}\left(\Phi^{(n+1)} * \Phi^{(n)}\right)=b_{0}\left(\Phi^{(n)} * \Phi^{(n)}\right)
$$

for $n=0,1,2, \cdots$, iteratively. If $\Phi^{(n)}$ converges to a string field with $n \rightarrow \infty, \Phi^{(\infty)}$ is a solution to (2.5). Actually, we stop the calculation when $\left\|\Phi^{(n+1)}-\Phi^{(n)}\right\| /\left\|\Phi^{(n)}\right\|<\varepsilon$ for a sufficiently small positive constant $\varepsilon$, where $\|\cdot\|$ is a norm and we regard $\Phi^{(n+1)}$ as an approximate solution.

We adopt the level truncation method to take a finite number of component fields from a string field $\Phi$ for numerical calculation. As a consistent level $L$ truncation, where $L$ is the eigenvalue of $L_{0}^{\text {mat }}+L_{0}^{\text {gh }}$, we expand a string field of the ghost number one with a basis which consists of twist even and $\mathrm{SU}(1,1)$ singlet states of the form:

$$
\begin{aligned}
& L_{-n_{m}}^{\mathrm{mat}} \cdots L_{-n_{1}}^{\mathrm{mat}} L_{-l_{g}}^{\mathrm{gh}} \cdots L_{-l_{1}}^{\mathrm{gh}} c_{1}|0\rangle, \\
& n_{m} \geq \cdots \geq n_{1} \geq 2, \quad l_{g} \geq \cdots \geq l_{1} \geq 2, \quad \sum_{k=1}^{m} n_{k}+\sum_{k=1}^{g} l_{k}=\ell, \quad(\ell=0,2,4, \cdots, L) .
\end{aligned}
$$


The truncation level $L$ is an even integer from the twist even condition and $|0\rangle$ is the conformal vacuum. We denote a state of the above form as $\psi_{i}$ and we expand $\Phi$ as

$$
\Phi=\sum_{i=1}^{N_{L}} t_{i} \psi_{i}
$$

with coefficients $t_{i}$, where $N_{L}$ is the dimension of the truncated state space up to level $L$, namely, $N_{L}=1,3,8,21,51,117, \cdots$ for $L=0,2,4,6,8,10, \cdots$, respectively. We take $t_{i}$ as a complex constant in general although it should be real from the reality condition of string fields. It is necessary to construct "double brane" and "ghost brane" solutions as we will see later.

With the above level truncation of string fields, we take a BPZ inner product of $c_{0} \psi_{i}$ and (2.8) and we obtain simultaneous equations:

$$
\sum_{j=1}^{N_{L}}\left((L(a))_{i j}+2 \sum_{k=1}^{N_{L}} V_{i j k} t_{k}^{(n)}\right) t_{j}^{(n+1)}=\sum_{j, k=1}^{N_{L}} V_{i j k} t_{k}^{(n)} t_{j}^{(n)},
$$

where

$$
(L(a))_{i j}=\left\langle\psi_{i}, c_{0} L(a) \psi_{j}\right\rangle, \quad V_{i j k}=\left\langle\psi_{i}, \psi_{j} * \psi_{k}\right\rangle,
$$

and use has been made of $V_{i j k}=V_{i k j}$ thanks to the twist even condition. With appropriate initial values $\left\{t_{i}^{(0)}\right\}_{i=1,2, \cdots, N_{L}}\left(t_{i}^{(0)} \in \mathbb{C}\right)$, we solve (2.12) iteratively for $n=0,1,2, \cdots$. We stop the calculation if $\left\|\boldsymbol{t}^{(n+1)}-\boldsymbol{t}^{(n)}\right\| /\left\|\boldsymbol{t}^{(n)}\right\|<\varepsilon$ with the Euclidean norm for a sufficiently small positive $\varepsilon$ and we regard

$$
\Phi_{a}=\sum_{i=1}^{N_{L}} \tilde{t}_{i} \psi_{i}
$$

$\left(\tilde{t}_{i}=t_{i}^{(n+1)}\right)$ as a numerical solution to $(2.5) .^{4}$

For a numerical solution $\Phi_{a}$, we compute the energy $E\left[\Phi_{a}\right]$, which is given by the action (2.1) as

$$
E\left[\Phi_{a}\right]=1-2 \pi^{2} g^{2} S_{a}\left[\Phi_{a}\right]=1+\frac{\pi^{2}}{3} \sum_{i, j=1}^{N_{L}}(L(a))_{i j} \tilde{t}_{i} \tilde{t}_{j},
$$

where we have used the equation of motion for $\tilde{\boldsymbol{t}}$ :

$$
\sum_{j=1}^{N_{L}}(L(a))_{i j} \tilde{t}_{j}+\sum_{j, k=1}^{N_{L}} V_{i j k} \tilde{t}_{k} \tilde{t}_{j}=0 .
$$

$E\left[\Phi_{a}\right](2.15)$ is normalized in the same way as [5]. In the case $a=0$, or in the theory around the perturbative vacuum, $\left.E[0]\right|_{a=0}=1$ for the single brane solution 0 , which is the perturbative vacuum, and $\left.E\left[\Psi^{\mathrm{T}}\right]\right|_{a=0}=0$ for the tachyon vacuum solution $\Psi^{\mathrm{T}}$.

\footnotetext{
${ }^{4}$ In our actual calculation in section 3 and section 4 , we took $\varepsilon=5 \times 10^{-14}$ in our $\mathrm{C}++$ code with the long double format. If $\left\|\boldsymbol{t}^{(n+1)}-\boldsymbol{t}^{(n)}\right\| /\left\|\boldsymbol{t}^{(n)}\right\| \geq \varepsilon$ for $n+1=15$, we regarded it as not converging.
} 
We evaluate the gauge invariant observable $E_{0}\left[\Phi_{a}\right]$ for $\Phi_{a}$ :

$$
E_{0}\left[\Phi_{a}\right]=1-2 \pi\left\langle I|V| \Phi_{a}\right\rangle
$$

where $V$ is given by $c(i) c(-i) \mathcal{V}(i,-i)$ and $\mathcal{V}(z, \bar{z})$ is matter primary with conformal weight $(1,1)$ and is normalized as

$$
\left\langle I\left|V c_{1}\right| 0\right\rangle=\frac{1}{4}
$$

We note that $(2.17)$ satisfies $E_{0}\left[\Psi_{\mathrm{Sch}}\right]=0[13,14]$ for Schnabl's analytic solution for tachyon condensation $\Psi_{\text {Sch }}[15]$.

For the tachyon vacuum solution $\Phi_{a}^{\mathrm{T}}$ and the perturbative vacuum (or single brane) solution $\Phi_{a}^{\mathrm{S}}$, in the theory around the TT solution $\Psi_{a}^{\mathrm{TT}}(a \geq-1 / 2)$, it has been shown that $E(2.15)$ and $E_{0}(2.17)$ behave as

$$
\begin{aligned}
& E\left[\Phi_{a}^{\mathrm{T}}\right] \rightarrow\left\{\begin{array}{ll}
0 & (a>-1 / 2) \\
1 & (a=-1 / 2)
\end{array}, \quad E_{0}\left[\Phi_{a}^{\mathrm{T}}\right] \rightarrow\left\{\begin{array}{ll}
0 & (a>-1 / 2) \\
1 & (a=-1 / 2)
\end{array},\right.\right. \\
& E\left[\Phi_{a}^{\mathrm{S}}\right] \rightarrow\left\{\begin{array}{ll}
1 & (a>-1 / 2) \\
2 & (a=-1 / 2)
\end{array}, \quad E_{0}\left[\Phi_{a}^{\mathrm{S}}\right] \rightarrow\left\{\begin{array}{ll}
1 & (a>-1 / 2) \\
2 & (a=-1 / 2)
\end{array},\right.\right.
\end{aligned}
$$

numerically in the large truncation level limit $L \rightarrow \infty$, where $\Phi_{a=-1 / 2}^{\mathrm{T}} \rightarrow 0$ and $\Phi_{a>-1 / 2}^{\mathrm{S}} \rightarrow 0[9]$.

As a consistency of the equation of motion (2.4) for numerical solutions to (2.5), we evaluate

$$
\left|\Delta_{S}\left[\Phi_{a}\right]\right|=\left|\left\langle 0\left|c_{-1} b_{2} c_{0}\right| Q^{\prime} \Phi_{a}+\Phi_{a} * \Phi_{a}\right\rangle\right|
$$

after [5]. It is the lowest level verification of BRST invariance for Siegel gauge solutions in the context of [16]. Furthermore, we evaluate

$$
\operatorname{Im} / \operatorname{Re}\left[\Phi_{a}\right]=\frac{\|\operatorname{Im} \tilde{\boldsymbol{t}}\|}{\|\operatorname{Re} \tilde{\boldsymbol{t}}\|}
$$

for reality of numerical solutions, which is given by the ratio of the Euclidean norm of imaginary and real part of $\tilde{\boldsymbol{t}}$.

\section{3 "Double brane" solution}

In the theory around the perturbative vacuum, which is the case $a=0$ in (2.1), a "double brane" solution $\Phi_{a=0}^{\mathrm{D}}$ is obtained from one of complex solutions at the truncation level 2 . Taking a solution $\Phi_{a=0}^{\mathrm{D}}$ at level $L$ as an initial string field, we can obtain $\Phi_{a=0}^{\mathrm{D}}$ at level $L+2$ by Newton's method as explained in section 2 . Such a solution $\Phi_{a=0}^{\mathrm{D}}$ coincides with the "double brane" solution in [5].

In the case $a \neq 0$, we adopt a strategy to construct solutions $\Phi_{a}^{\mathrm{D}}$ corresponding to "double brane" as follows: 
1. At level 2, we construct a solution $\Phi_{a \mp \epsilon}^{\mathrm{D}}$ using Newton's method with an initial string field $\Phi_{a}^{\mathrm{D}}$. We repeat this calculation with $\epsilon=0.001$ up to $\Phi_{a=\mp 1 / 2}^{\mathrm{D}}$ from the starting point $\Phi_{a=0}^{\mathrm{D}}$.

2. At level $L+2$, we construct a solution $\Phi_{a}^{\mathrm{D}}$ using Newton's method with an initial string field $\Phi_{a}^{\mathrm{D}}$ at level $L$. We repeat this calculation up to level 22 .

Namely, for a fixed value of $a$, which is one of $a=-0.5,-0.499,-0.498, \cdots, 0.499,0.5$, we constructed higher level solutions $\Phi_{a}^{\mathrm{D}}$ from level 2 up to 22 , level by level.

Then, we have obtained numerical solutions $\Phi_{a}^{\mathrm{D}}$, except for $\Phi_{a=-0.468}^{\mathrm{D}}$ at levels 20 and 22. In the case $a=-0.468$, Newton's method for getting a solution at level 20 did not converge.

\subsection{Energy}

Figures 1, 2, and 3 show plots of the energy $E$ (2.15) for the "double brane" solution $\Phi_{a}^{\mathrm{D}}$ at the truncation level $L$. We joined adjacent calculated data points with line segments for each level. ${ }^{5}$ In figure 1 , the dotted and dashed lines are extrapolations to $L=4 k+2$ $(k \rightarrow \infty)$ and $L=4 k(k \rightarrow \infty)$, respectively. We have used a polynomial of $1 / L$ as a fitting function for each value of the parameter $a$. We have chosen its degree as the number of data minus one. ${ }^{6}$ As might be expected from figure 2 , extrapolations by a polynomial fit worked well by dividing data in two groups: $L \equiv 2 \bmod 4$ and $L \equiv 0 \bmod 4$.

As in figure 1, the real part of energy $\operatorname{Re} E\left[\Phi_{a}^{\mathrm{D}}\right]$ approaches a constant, which is greater than one, for $a>-1 / 2$ with increasing level and there is a maximum at $a=a_{\mathrm{M}} \sim-1 / 2$ for each $L$. As in figure 2 , the value of $a_{\mathrm{M}}$ approaches $-1 / 2$ with increasing level, where the maximum value is greater than two. The extrapolation values of $\operatorname{Re} E\left[\Phi_{a}^{\mathrm{D}}\right]$ in figure 1 are close to 1.5 for $a>-1 / 2$.

As in figure 3, the imaginary part of energy $\operatorname{Im} E\left[\Phi_{a}^{\mathrm{D}}\right]$ approaches zero for $a>-1 / 2$ with increasing level and there is a minimum at $a=a_{\mathrm{m}} \sim-1 / 2$ for each $L$. The extrapolation values of $\operatorname{Im} E\left[\Phi_{a}^{\mathrm{D}}\right]$ in figure 3 are close to zero for $a>-1 / 2$. We observed that $a_{\mathrm{m}}$ approaches $-1 / 2$ and the minimum value approaches zero with increasing level although extrapolation values are unstable near $a=-1 / 2$.

From the above observation and analogy with tachyon vacuum and single brane solutions in (2.19) and (2.20), we could expect that the energy (2.15) for the "double brane" solution $\Phi_{a}^{\mathrm{D}}$ behaves as

$$
E\left[\Phi_{a}^{\mathrm{D}}\right] \rightarrow\left\{\begin{array}{ll}
E_{2} & (a>-1 / 2) \\
E_{2}^{\prime} & (a=-1 / 2)
\end{array} \quad(L \rightarrow \infty),\right.
$$

where $E_{2}$ and $E_{2}^{\prime}$ seem to be real constants and satisfy $1<E_{2}<E_{2}^{\prime}$. If $E_{2}=2$ and $E_{2}^{\prime}=3$, $\Phi_{a}^{\mathrm{D}}$ could be interpreted as double brane solution for $a \geq-1 / 2$ literally. However, it seems that $E_{2} \sim 1.5$ and $2<E_{2}^{\prime}<3$ from figures 1 and 2 . If $E_{2}^{\prime}-E_{2}=1$, the solution $\Phi_{a}^{\mathrm{D}}$ for $a \geq-1 / 2$ is consistent with the interpretation that the TT solution $\Psi_{a}^{\mathrm{TT}}$ represents

\footnotetext{
${ }^{5}$ Plots of other figures in this paper are in the same manner.

${ }^{6}$ Extrapolations in other figures in this paper have been obtained in the same manner.
} 


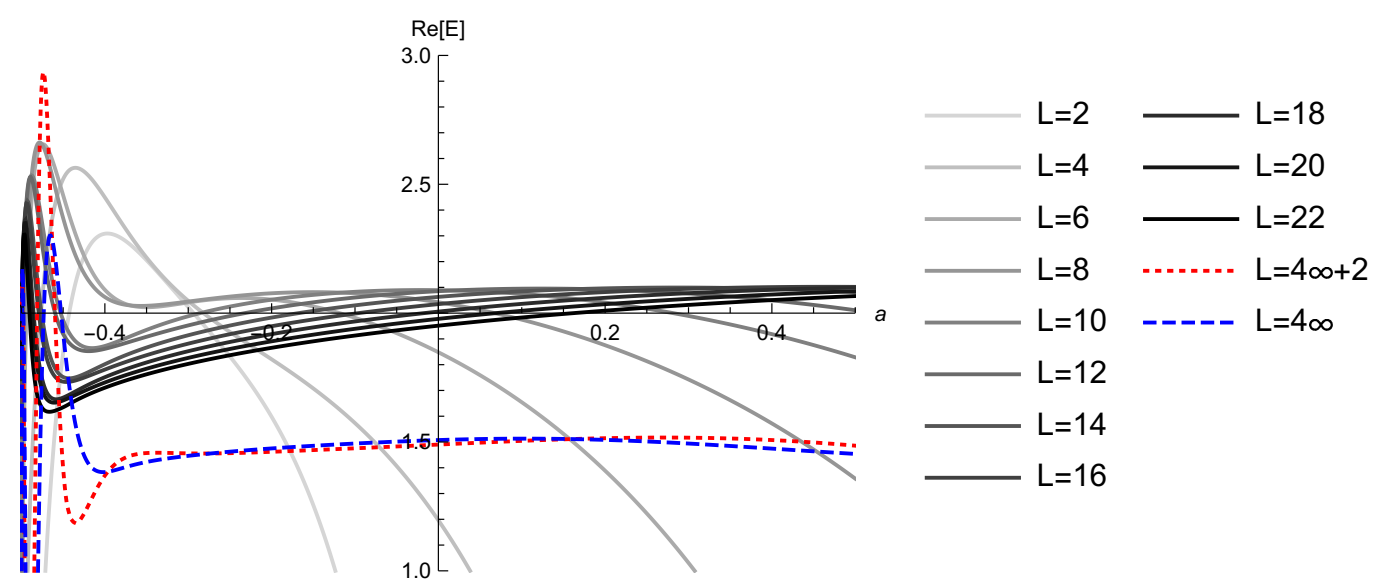

Figure 1. Plots of the real part of the energy $E(2.15)$ for the "double brane" solution $\Phi_{a}^{\mathrm{D}}$ at the truncation level $L$. The dotted and dashed lines are extrapolations to $L=4 k+2(k \rightarrow \infty)$ and $L=4 k(k \rightarrow \infty)$, respectively. The horizontal axis denotes the value of the parameter $a$ at $\operatorname{Re} E=2$.

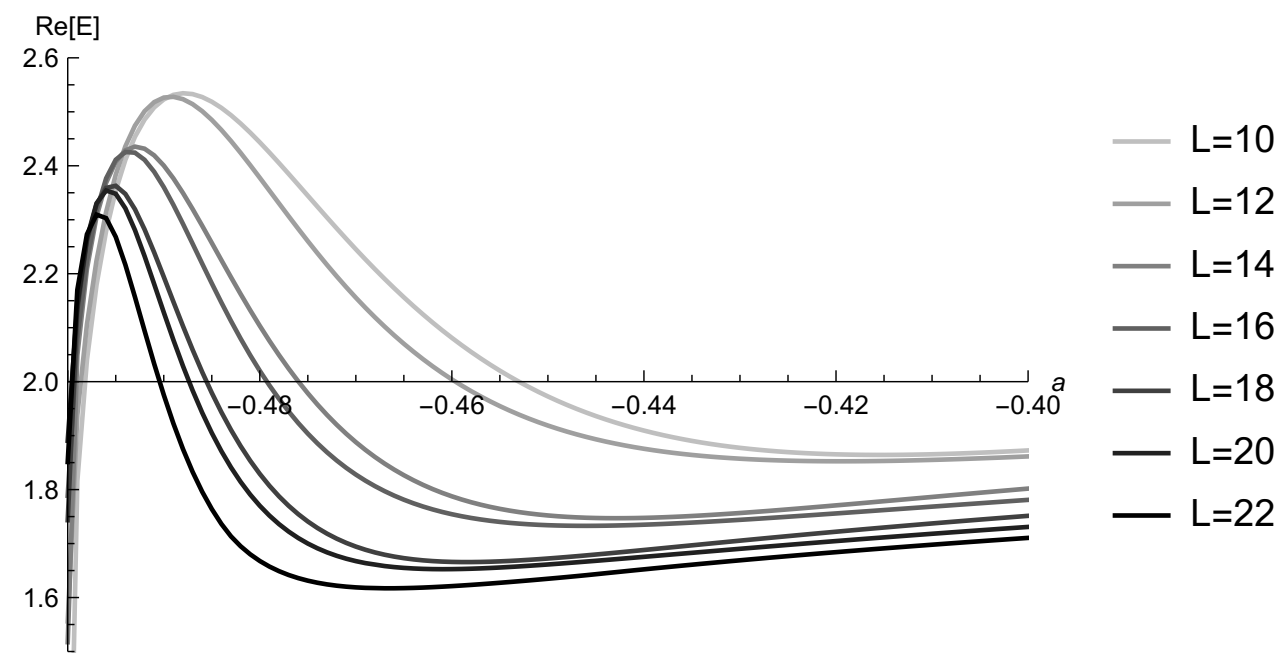

Figure 2. Plots of the real part of the energy $E(2.15)$ for the "double brane" solution $\Phi_{a}^{\mathrm{D}}$ for $a \gtrsim-1 / 2$ at the truncation level $L=10,12, \cdots, 22$. The horizontal axis denotes the value of the parameter $a$ at $\operatorname{Re} E=2$. The vertical axis stands at $a=-1 / 2$.

the tachyon vacuum at $a=-1 / 2$ and pure gauge solution for $a>-1 / 2$, which implies $S\left[\Psi_{a=-1 / 2}^{\mathrm{TT}}\right]=1 /\left(2 \pi^{2} g^{2}\right)$ and $S\left[\Psi_{a>-1 / 2}^{\mathrm{TT}}\right]=0$.

\subsection{Gauge invariant observable}

Figures 4,5 , and 6 show plots of the gauge invariant observable $E_{0}(2.17)$ for the "double brane" solution $\Phi_{a}^{\mathrm{D}}$ at the truncation level $L$.

As in figure 4 , the real part $\operatorname{Re} E_{0}\left[\Phi_{a}^{\mathrm{D}}\right]$ approaches a constant, which is greater than one, for $a>-1 / 2$ with increasing level. As in figure 5 (and table 1 in appendix A), $\operatorname{Re} E_{0}\left[\Phi_{a}^{\mathrm{D}}\right]>2$ for $a \rightarrow-1 / 2+0$. The extrapolation values of $\operatorname{Re} E_{0}\left[\Phi_{a}^{\mathrm{D}}\right]$ in figure 4 are 


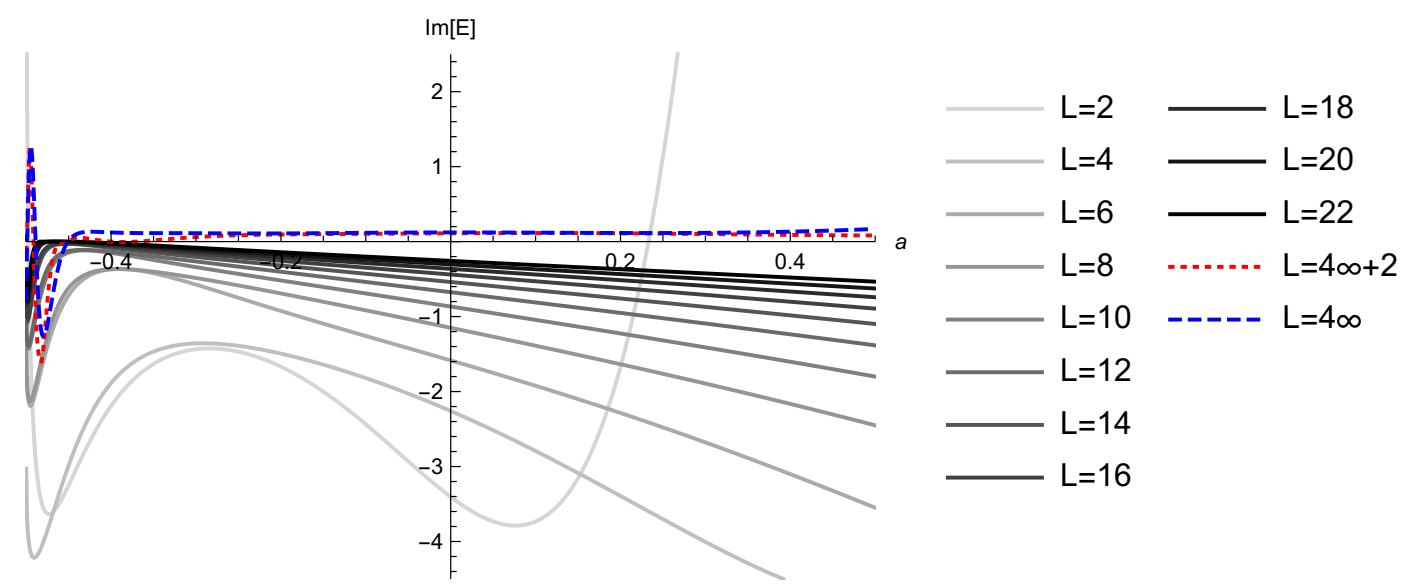

Figure 3. Plots of the imaginary part of the energy $E(2.15)$ for the "double brane" solution $\Phi_{a}^{\mathrm{D}}$ at the truncation level $L$. The dotted and dashed lines are extrapolations to $L=4 k+2(k \rightarrow \infty)$ and $L=4 k(k \rightarrow \infty)$, respectively. The horizontal axis denotes the value of the parameter $a$.

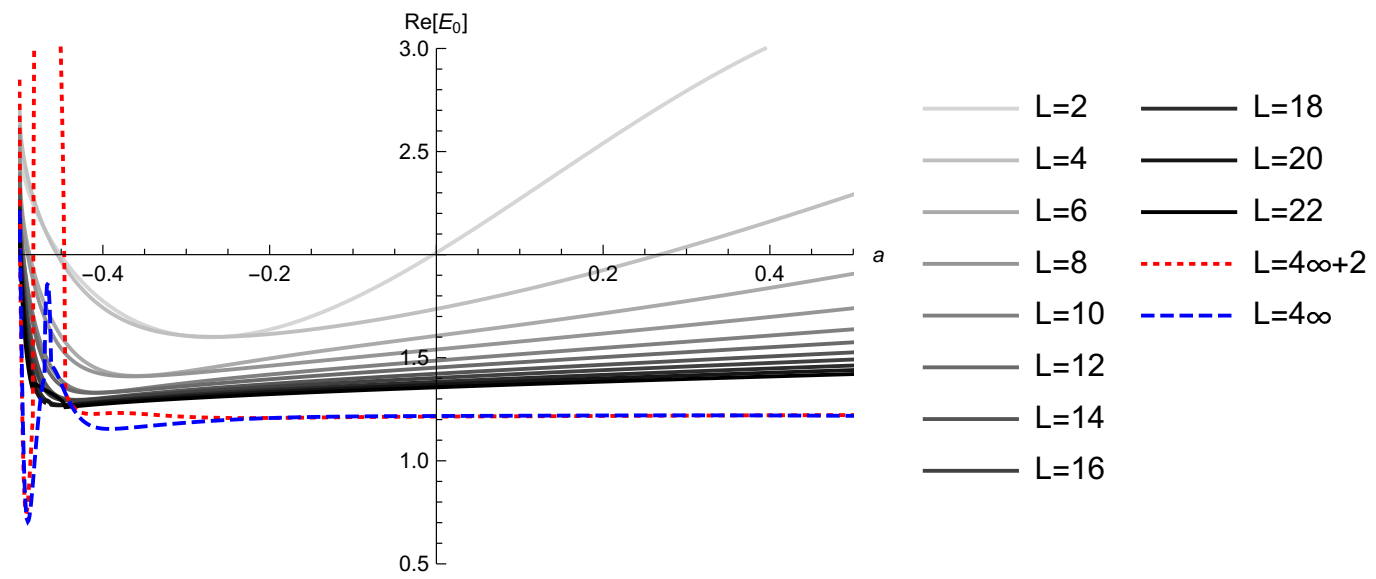

Figure 4. Plots of the real part of the gauge invariant observable $E_{0}(2.17)$ for the "double brane" solution $\Phi_{a}^{\mathrm{D}}$ at the truncation level $L$. The dotted and dashed lines are extrapolations to $L=4 k+2$ $(k \rightarrow \infty)$ and $L=4 k(k \rightarrow \infty)$, respectively. The horizontal axis denotes the value of the parameter $a$ at $\operatorname{Re} E_{0}=2$.

close to 1.2 for $a>-1 / 2$ although they become unstable near $a=-1 / 2$. This instability or error should be caused by irregular behavior around $a=-0.465$ at $L=20,22$ in figure 5 .

As in figure 6 , the imaginary part $\operatorname{Im} E_{0}\left[\Phi_{a}^{\mathrm{D}}\right]$ approaches zero for $a>-1 / 2$ with increasing level. The extrapolation values are around zero for $a>-1 / 2$ although they are unstable near $a=-1 / 2$. Actually, we have found that $\operatorname{Im} E_{0}\left[\Phi_{a}^{\mathrm{D}}\right]=0$ around $a=-0.465$ for $L=20,22$.

From the above, in a similar way to (3.1), we could expect that the gauge invariant observable (2.17) behaves as

$$
E_{0}\left[\Phi_{a}^{\mathrm{D}}\right] \rightarrow\left\{\begin{array}{ll}
\tilde{E}_{2} & (a>-1 / 2) \\
\tilde{E}_{2}^{\prime} & (a=-1 / 2)
\end{array} \quad(L \rightarrow \infty),\right.
$$




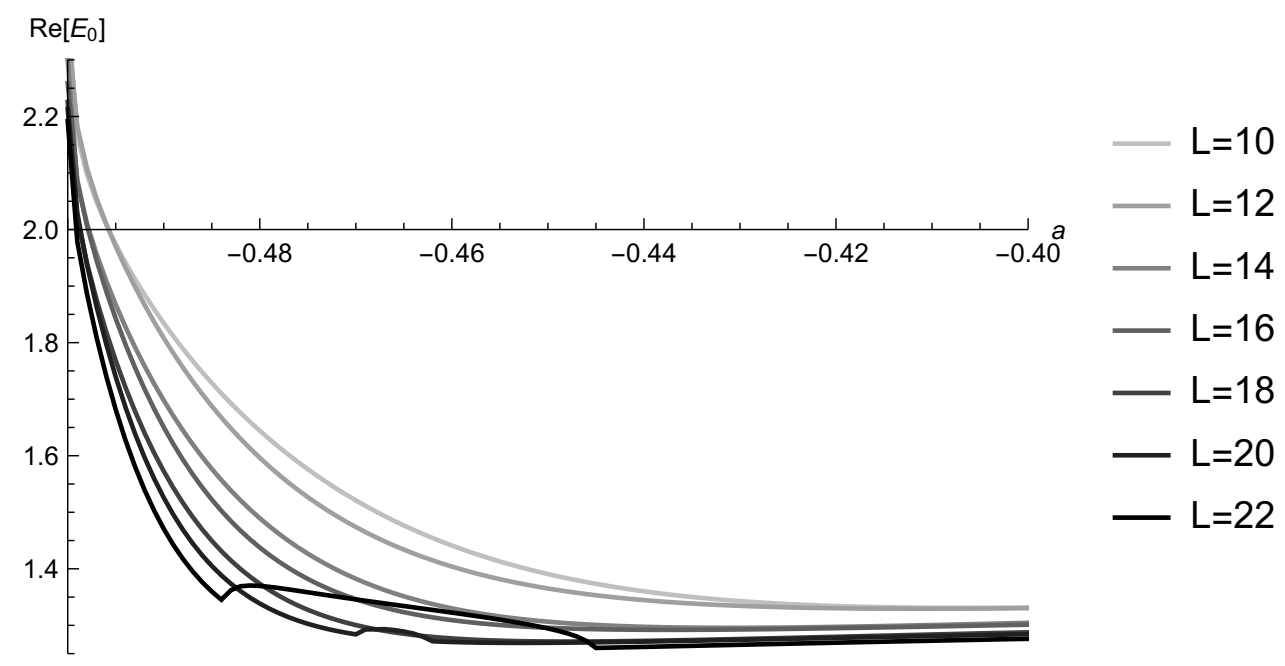

Figure 5. Plots of the real part of the gauge invariant observable $E_{0}(2.17)$ for the "double brane" solution $\Phi_{a}^{\mathrm{D}}$ such as $a \gtrsim-1 / 2$ at the truncation level $L=10,12, \cdots, 22$. The horizontal axis denotes the value of the parameter $a$ at $\operatorname{Re} E_{0}=2$. The vertical axis stands at $a=-1 / 2$.

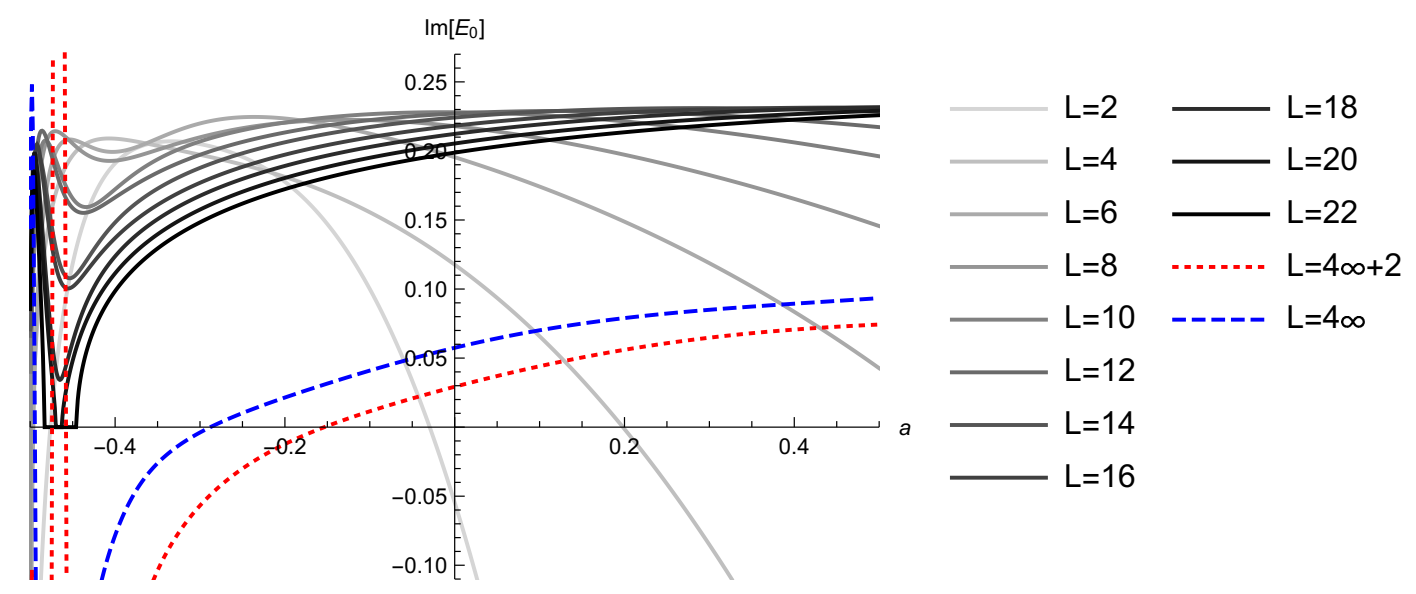

Figure 6. Plots of the imaginary part of the gauge invariant observable $E_{0}(2.17)$ for the "double brane" solution $\Phi_{a}^{\mathrm{D}}$ at the truncation level $L$. The dotted and dashed lines are extrapolations to $L=4 k+2(k \rightarrow \infty)$ and $L=4 k(k \rightarrow \infty)$, respectively. The horizontal axis denotes the value of the parameter $a$.

where $\tilde{E}_{2}$ and $\tilde{E}_{2}^{\prime}$ seem to be real constants and satisfy $1<\tilde{E}_{2}<\tilde{E}_{2}^{\prime}$. If $\tilde{E}_{2}=2$ and $\tilde{E}_{2}^{\prime}=3$, $\Phi_{a}^{\mathrm{D}}$ could be interpreted as double brane solution for $a \geq-1 / 2$ because the value of (2.17) corresponds to the energy [17]. However, it seems that $\tilde{E}_{2} \sim 1.2$ and $2 \lesssim \tilde{E}_{2}^{\prime}<3$ from figures 4 and 5. If $\tilde{E}_{2}^{\prime}-\tilde{E}_{2}=1$, the solution $\Phi_{a}^{\mathrm{D}}$ for $a>-1 / 2$ is consistent with the TT solution in the same sense as the energy.

\section{$3.3 \quad\left|\Delta_{S}\right|$ and reality}

Figure 7 shows plots of $\left|\Delta_{S}\right|$ (2.21) for the "double brane" solution $\Phi_{a}^{\mathrm{D}}$ at the truncation level $L$. With increasing level, it approaches zero for $a \geq-1 / 2$. This behavior is consistent 


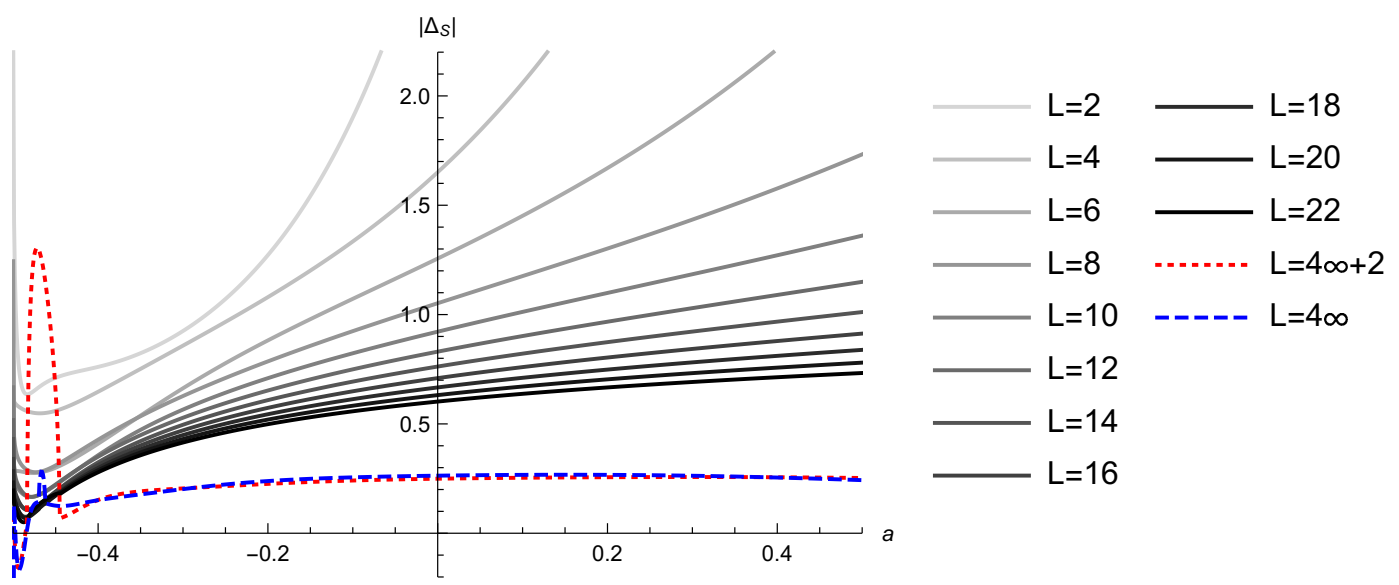

Figure 7. Plots of $\left|\Delta_{S}\right|(2.21)$ for the "double brane" solution $\Phi_{a}^{\mathrm{D}}$ at the truncation level $L$. The horizontal axis denotes the value of the parameter $a$.

with the equation of motion (2.4) for $\Phi_{a}^{\mathrm{D}}$. The extrapolation values are close to 0.25 for $a>-1 / 2$ except around $a=-1 / 2$, where they are unstable. We could expect that the above does not contradict that $\left|\Delta_{S}\right| \rightarrow 0(L \rightarrow \infty)$ for $a \geq-1 / 2$.

Figures 8 and 9 show plots of $\operatorname{Im} / \operatorname{Re}(2.22)$ for the "double brane" solution $\Phi_{a}^{\mathrm{D}}$ at the truncation level $L$. With increasing level, it approaches zero for $a \geq-1 / 2$. This behavior is consistent with the reality condition of the string field $\Phi_{a}^{\mathrm{D}}$. In particular, from figure 9 , we have found that $\operatorname{Im} / \operatorname{Re}=0$ for $-0.469 \leq a \leq-0.463$ at $L=20$ and for $-0.483 \leq a \leq-0.446$ at $L=22$ (although there is no solution at $a=-0.468$ in both cases as mentioned). The region of $a$ at $L=22$, where $\Phi_{a}^{\mathrm{D}}$ is real, is larger than that at $L=20$. We can expect that it becomes larger at higher truncation level because $\Phi_{a}^{\mathrm{D}}$ at the level $L+2$ is real once it becomes real at the level $L$ from our method to construct the solutions. We note that the regions of $a$ such that $\Phi_{a}^{\mathrm{D}}$ is real correspond to those where the value of $\operatorname{Re} E_{0}\left[\Phi_{a}^{\mathrm{D}}\right]$ shows irregular behavior in figure 5 .

In figure 8 , the extrapolation values become negative for some region of $a$ although $\mathrm{Im} /$ Re should be nonnegative by its definition (2.22). We might interpret that $\Phi_{a}^{\mathrm{D}}$ becomes real at finite level in such a region.

From the above observations, we expect that the "double brane" solution $\Phi_{a}^{\mathrm{D}}$ for $a \geq$ $-1 / 2$ satisfies the reality condition in the large level limit $L \rightarrow \infty$.

\section{4 "Ghost brane" solution}

Our strategy to construct solutions corresponding to "ghost brane" is the same as that in section 3.

In the theory around the perturbative vacuum, which is the case $a=0$ in (2.1), a "ghost brane" solution $\Phi_{a=0}^{\mathrm{G}}$ is obtained from one of complex solutions at the truncation level 4. Taking a solution $\Phi_{a=0}^{\mathrm{G}}$ at level $L$ as an initial string field, we can obtain $\Phi_{a=0}^{\mathrm{G}}$ at level $L+2$ by Newton's method. Such a solution $\Phi_{a=0}^{\mathrm{G}}$ coincides with the "ghost brane" solution in [5]. 


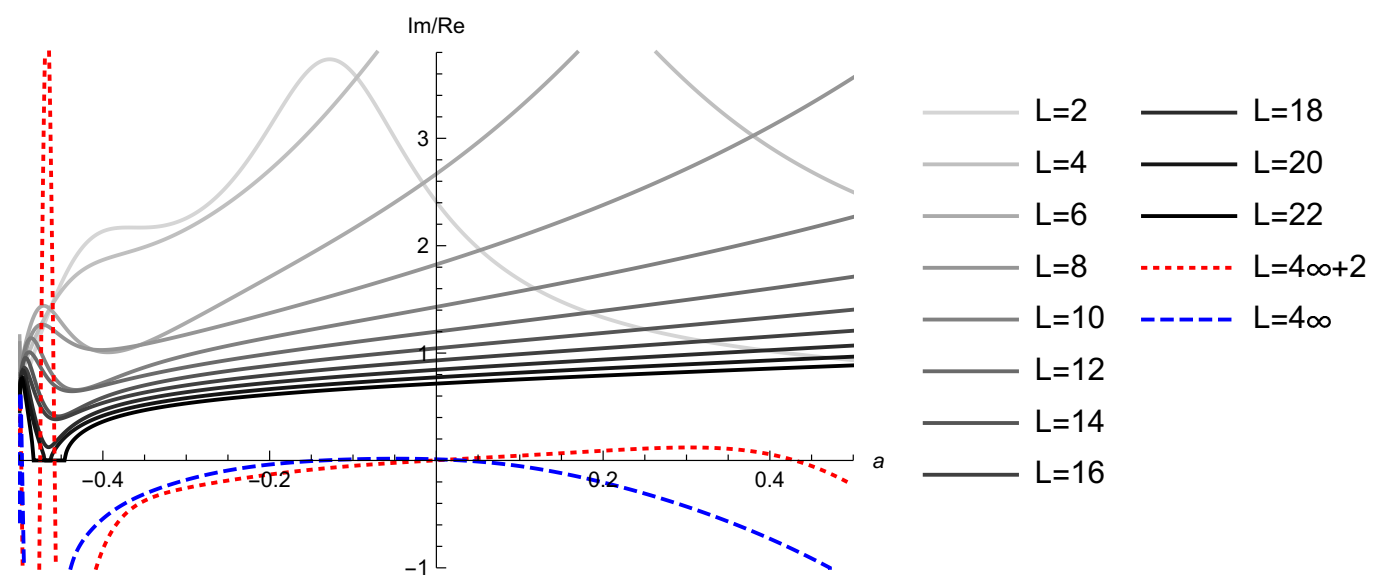

Figure 8. Plots of Im/Re (2.22) for the "double brane" solution $\Phi_{a}^{\mathrm{D}}$ at the truncation level $L$. The horizontal axis denotes the value of the parameter $a$.

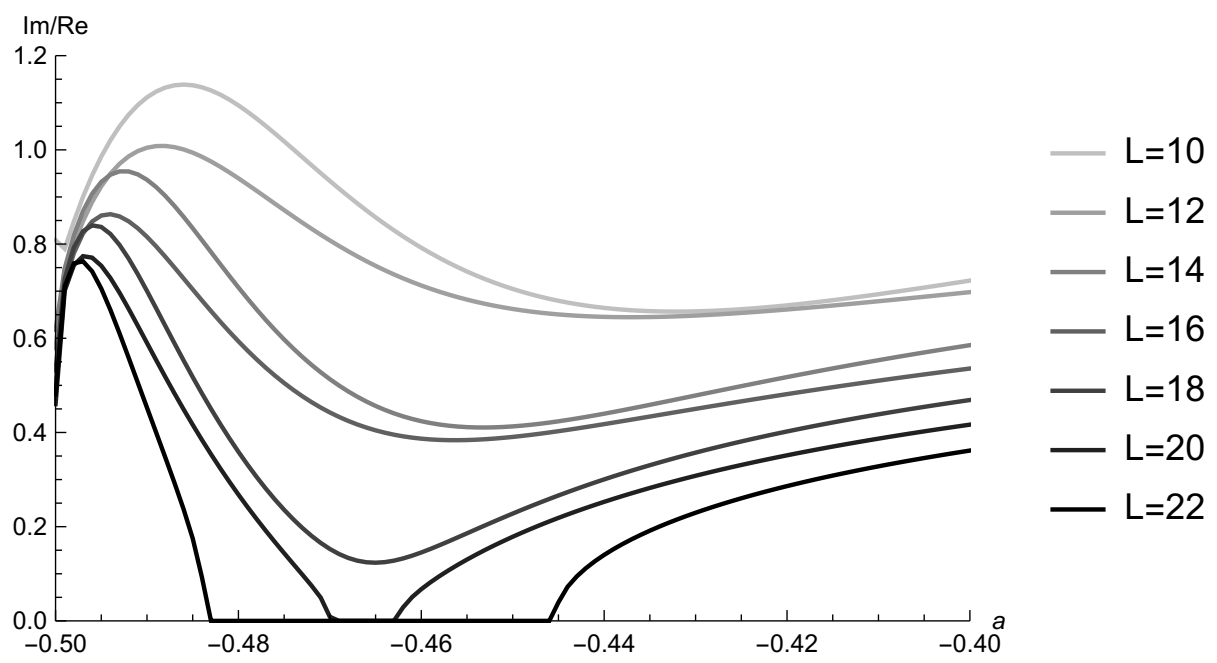

Figure 9. Plots of $\operatorname{Im} / \operatorname{Re}(2.22)$ for the "double brane" solution $\Phi_{a}^{\mathrm{D}}$ such as $a \gtrsim-1 / 2$ at the truncation level $L=10,12, \cdots, 22$. The horizontal axis denotes the value of the parameter $a$. The vertical axis stands at $a=-1 / 2$.

Firstly, at the truncation level 4 , we constructed $\Phi_{a}^{\mathrm{G}}(-1 / 2 \leq a \leq 1 / 2)$ from $\Phi_{a=0}^{\mathrm{G}}$. Then, for a fixed value of $a$, which is one of $a=-0.5,-0.499,-0.498, \cdots, 0.499,0.5$, we constructed higher level solutions from $\Phi_{a}^{\mathrm{G}}$ at level 4 up to 22. As a result, we have obtained numerical solutions $\Phi_{a}^{\mathrm{G}}$, except for $\Phi_{a=-0.499}^{\mathrm{G}}$ at level 22. In the case $a=-0.499$, Newton's method for constructing a solution at level 22 did not converge.

\subsection{Energy}

Figures 10 and 11 show plots of the energy $E$ (2.15) for the "ghost brane" solution $\Phi_{a}^{\mathrm{G}}$ at the truncation level $L$. As in figure 10 , the real part $\operatorname{Re} E\left[\Phi_{a}^{\mathrm{G}}\right]$ increases with increasing level. The extrapolations are close to -1 for $a>-1 / 2$. There is a maximum near $a=-1 / 2$ for each extrapolation and the maximum value is greater than zero. 


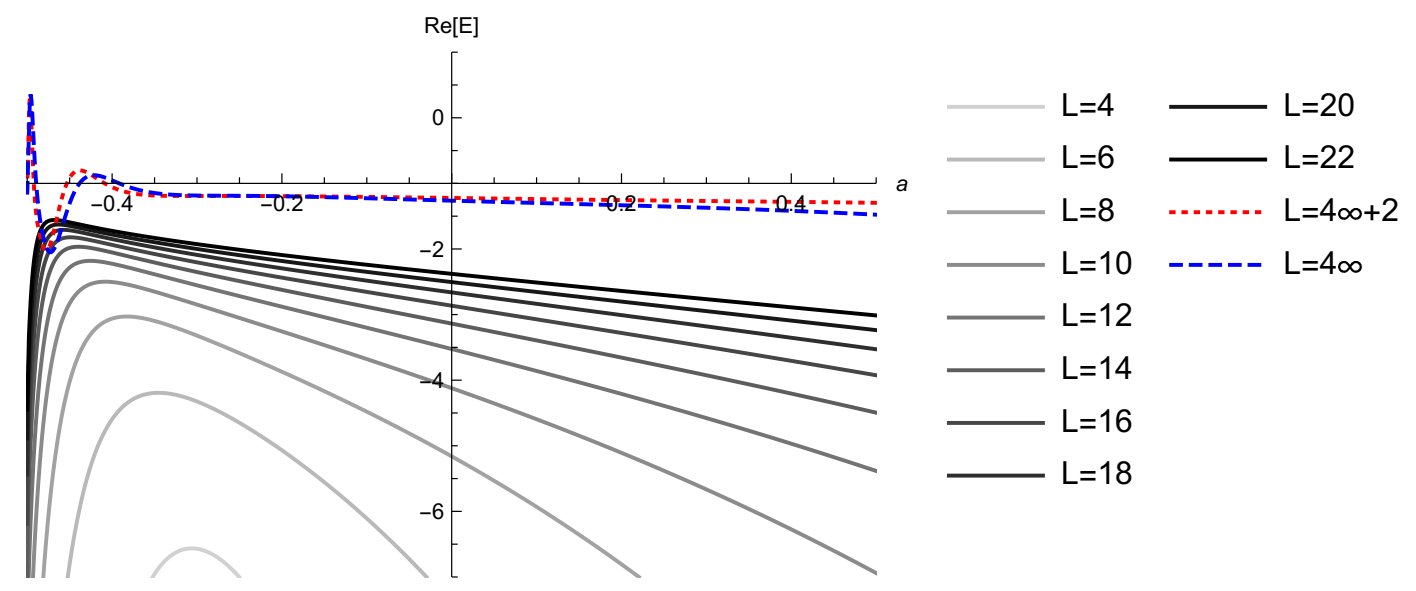

Figure 10. Plots of the real part of the energy $E(2.15)$ for the "ghost brane" solution $\Phi_{a}^{\mathrm{G}}$ at the truncation level $L$. The dotted and dashed lines are extrapolations to $L=4 k+2(k \rightarrow \infty)$ and $L=4 k(k \rightarrow \infty)$, respectively. The horizontal axis denotes the value of the parameter $a$ at Re $E=-1$.

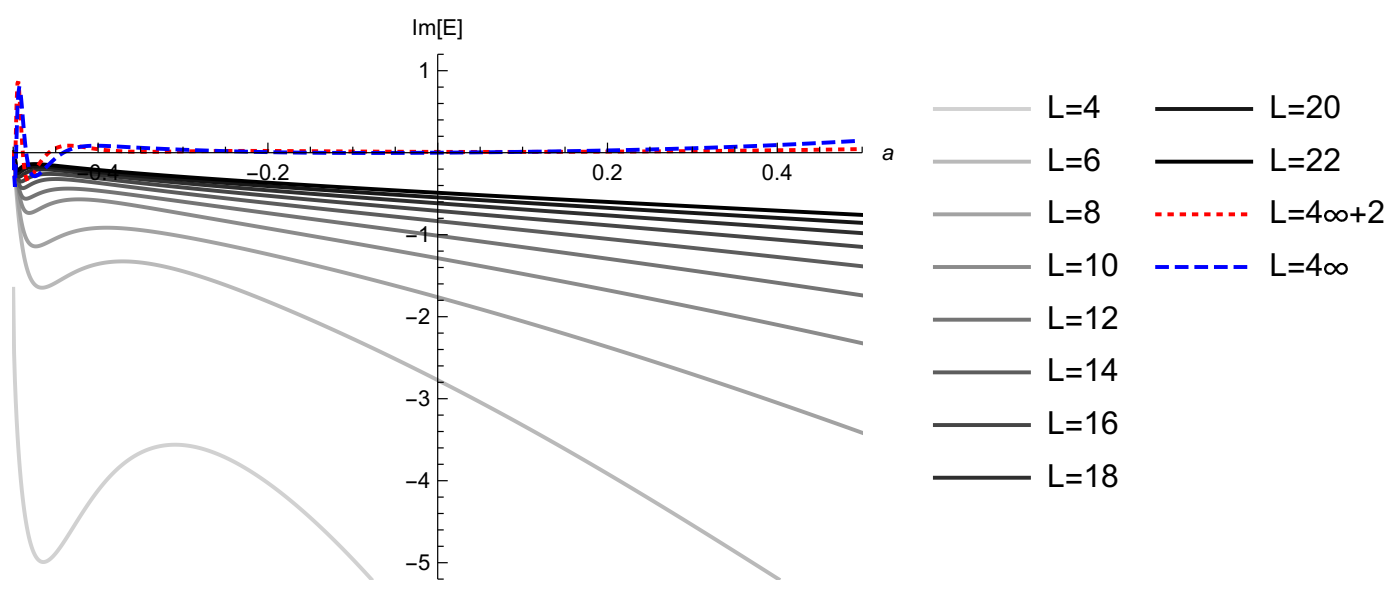

Figure 11. Plots of the imaginary part of the energy $E(2.15)$ for the "ghost brane" solution $\Phi_{a}^{\mathrm{G}}$ at the truncation level $L$. The dotted and dashed lines are extrapolations to $L=4 k+2(k \rightarrow \infty)$ and $L=4 k(k \rightarrow \infty)$, respectively. The horizontal axis denotes the value of the parameter $a$.

As in figure 11, the imaginary part $\operatorname{Im} E\left[\Phi_{a}^{\mathrm{G}}\right]$ approaches zero with increasing level. In particular, extrapolation values are close to zero for $a>-1 / 2$ although they become unstable around $a=-1 / 2$. We note that $\operatorname{Im} E\left[\Phi_{a}^{\mathrm{G}}\right]=0$ at $a=-1 / 2$ for the level $L \geq 6$ as in table 2 in appendix A.

From the above, we might expect that the energy for the "ghost brane" solution behaves as

$$
E\left[\Phi_{a}^{\mathrm{G}}\right] \rightarrow\left\{\begin{array}{ll}
E_{-1} & (a>-1 / 2) \\
E_{-1}^{\prime} & (a=-1 / 2)
\end{array} \quad(L \rightarrow \infty),\right.
$$

where $E_{-1}$ and $E_{-1}^{\prime}$ seem to be real constants such as $-2<E_{-1}<E_{-1}^{\prime}$, in a similar way to (3.1) for $\Phi_{a}^{\mathrm{D}}$, but it is more obscure in this case. If we focus on the extrapolation values, 


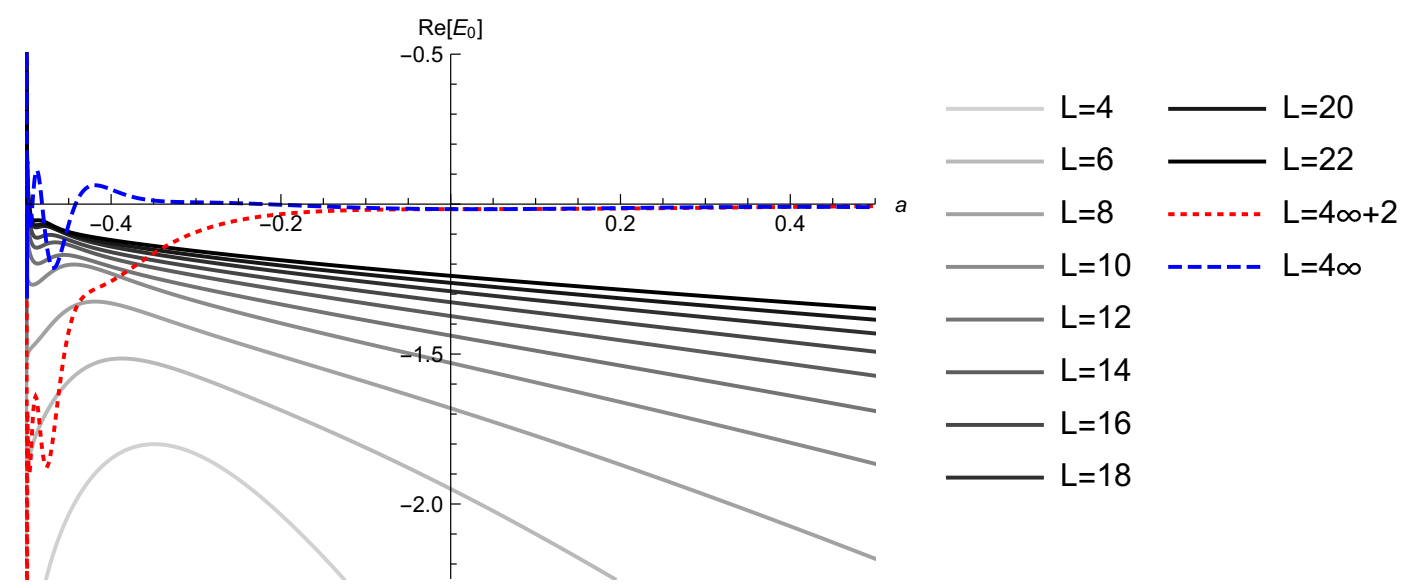

Figure 12. Plots of the real part of the gauge invariant observable $E_{0}(2.17)$ for the "ghost brane" solution $\Phi_{a}^{\mathrm{G}}$ at the truncation level $L$. The dotted and dashed lines are extrapolations to $L=4 k+2$ $(k \rightarrow \infty)$ and $L=4 k(k \rightarrow \infty)$, respectively. The horizontal axis denotes the value of the parameter $a$ at $\operatorname{Re} E_{0}=-1$.

it seems that $E_{-1} \sim-1.25$ and $0<E_{-1}^{\prime}<1$, although $\Phi_{a}^{\mathrm{G}}$ could represent ghost brane literally if $E_{-1}=-1$ and $E_{-1}^{\prime}=0$. If $E_{-1}^{\prime}-E_{-1}=1, \Phi_{a}^{\mathrm{G}}$ for $a \geq-1 / 2$ is consistent with the $a$-dependence of the TT solution.

\subsection{Gauge invariant observable}

Figures 12, 13, and 14 show plots of the gauge invariant observable $E_{0}(2.17)$ for the "ghost brane" solution $\Phi_{a}^{\mathrm{G}}$ at the truncation level $L$. As in figure 12 , the real part $\operatorname{Re} E_{0}\left[\Phi_{a}^{\mathrm{G}}\right]$ approaches -1 for $a>-1 / 2$. As in figure 13 , there is a maximum near $a=-1 / 2$ for each level $L$. We have found that the maximum values are greater than -1 for $L \geq 10$ although there is a large error in extrapolation values of $\operatorname{Re} E_{0}\left[\Phi_{a}^{\mathrm{G}}\right]$ near $a=-1 / 2$ in figure $12 .^{7}$

As in figure 14, the imaginary part $\operatorname{Im} E_{0}\left[\Phi_{a}^{\mathrm{G}}\right]$ decreases with increasing level. Its extrapolation values are about 0.1 for $a>-1 / 2$ and they become unstable around $a=$ $-1 / 2$. At $a=-1 / 2, \operatorname{Im} E_{0}\left[\Phi_{a}^{\mathrm{G}}\right]=0$ for the level $L \geq 6$ as in table 2 in appendix A.

From the above, in a similar way to (4.1), we could expect that the gauge invariant observable (2.17) behaves as

$$
E_{0}\left[\Phi_{a}^{\mathrm{G}}\right] \rightarrow\left\{\begin{array}{ll}
\tilde{E}_{-1} & (a>-1 / 2) \\
\tilde{E}_{-1}^{\prime} & (a=-1 / 2)
\end{array} \quad(L \rightarrow \infty)\right.
$$

where $\tilde{E}_{-1}$ and $\tilde{E}_{-1}^{\prime}$ seem to be real constants such as $-2<\tilde{E}_{-1}<\tilde{E}_{-1}^{\prime}$.

\section{3 $\left|\Delta_{S}\right|$ and reality}

Figure 15 shows plots of $\left|\Delta_{S}\right|(2.21)$ for the "ghost brane" solution $\Phi_{a}^{\mathrm{G}}$ at the truncation level $L$. It decreases with increasing level. The extrapolation values are about 0.1 for

\footnotetext{
${ }^{7}$ We performed computations at $a=-0.5,-0.4999,-0.4998, \cdots,-0.4901,-0.49$ in order to show details near $a=-0.5$ in figure 13 (and figure 12). In addition to $a=-0.499$ for $L=22$, we did not obtain solutions at $a=-0.4989$ for $L=20,22$ and at $a=-0.4988$ for $L=16,18,20,22$.
} 


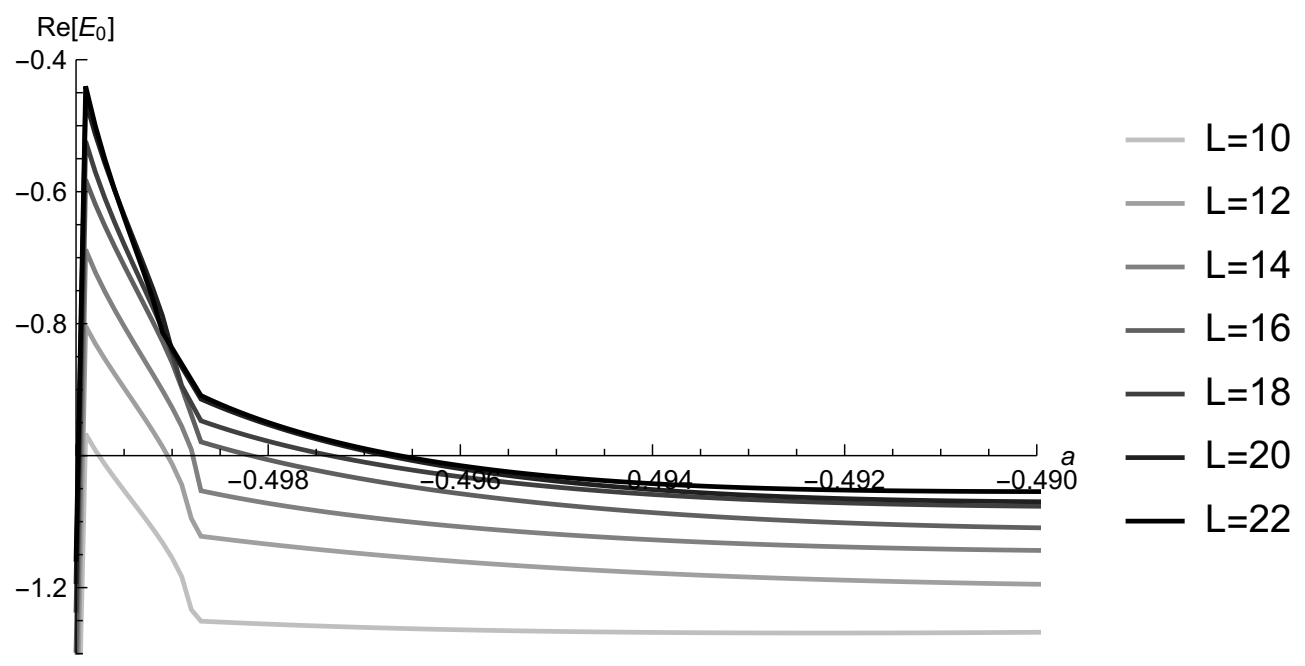

Figure 13. Plots of the real part of the gauge invariant observable $E_{0}(2.17)$ for the "ghost brane" solution $\Phi_{a}^{\mathrm{G}}$ near $a=-1 / 2$ at the truncation level $L=10,12, \cdots, 22$. The horizontal axis denotes the value of the parameter $a$ at $\operatorname{Re} E_{0}=-1$. The vertical axis stands at $a=-1 / 2$.

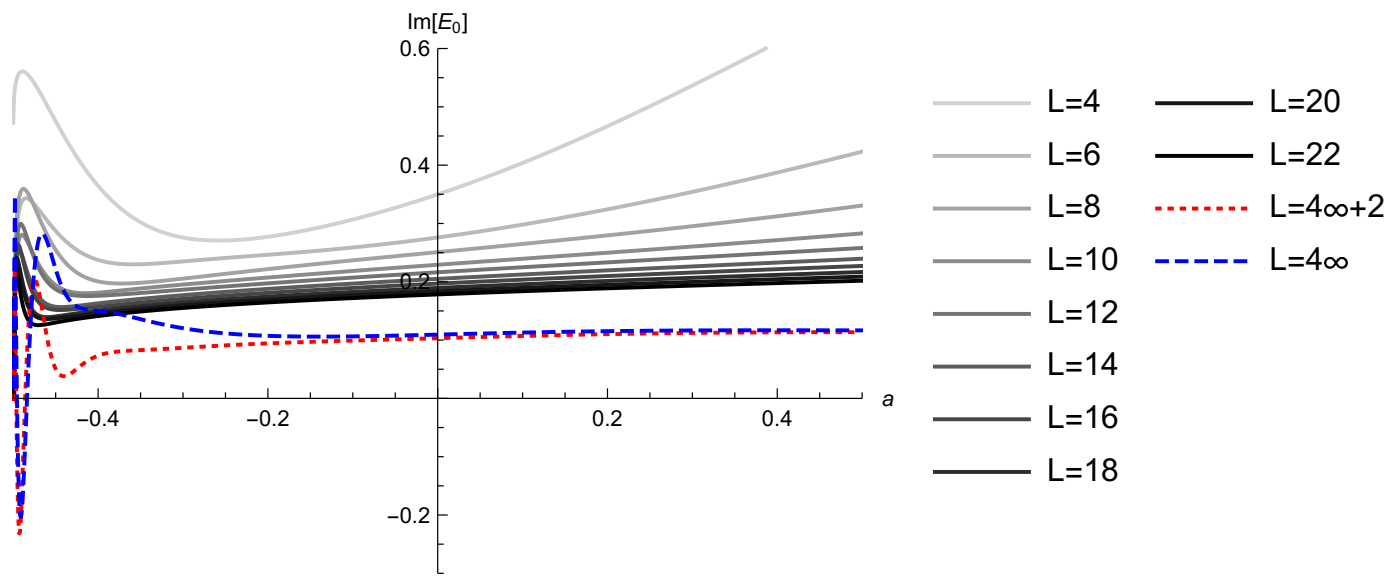

Figure 14. Plots of the imaginary part of the gauge invariant observable $E_{0}(2.17)$ for the "ghost brane" solution $\Phi_{a}^{\mathrm{G}}$ at the truncation level $L$. The dotted and dashed lines are extrapolations to $L=4 k+2(k \rightarrow \infty)$ and $L=4 k(k \rightarrow \infty)$, respectively. The horizontal axis denotes the value of the parameter $a$.

$a \gtrsim-1 / 2$ and they are close to zero near $a=-1 / 2$. It is consistent with the equation of motion (2.4) for $\Phi_{a}^{\mathrm{G}}$.

Figure 16 shows plots of $\operatorname{Im} / \operatorname{Re}(2.22)$ for the "ghost brane" solution $\Phi_{a}^{\mathrm{G}}$ at the truncation level $L$. For $a \gtrsim-0.4, \mathrm{Im} /$ Re at level $L$ up to 22 and its extrapolation values are greater than 0.2. On the other hand, we found that $\operatorname{Im} / \operatorname{Re}\left[\Phi_{a}^{\mathrm{G}}\right]=0$ at $a=-0.5,-0.499$ for $L \geq 6$, although there is no solution at $a=-0.499$ for $L=22$.

In any case, it seems that $\Phi_{a}^{\mathrm{G}}$ does not satisfy the reality condition for $a \gtrsim-0.4$ even at the limit $L \rightarrow \infty$ from the numerical behavior of $\operatorname{Im} / \operatorname{Re}\left[\Phi_{a}^{\mathrm{G}}\right]$ in figure 16 and comparison with that for $\Phi_{a}^{\mathrm{D}}$ in figure 8. It is consistent with the interpretation of the "ghost brane" solution in [5], which corresponds to the case $a=0$. 


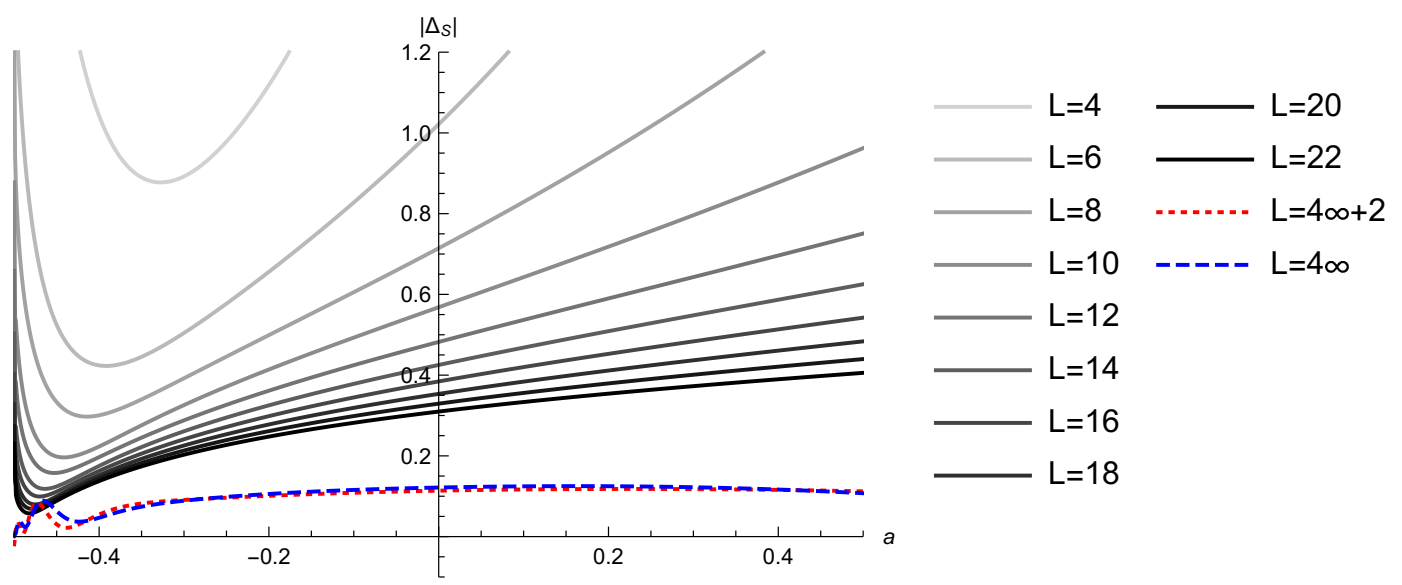

Figure 15. Plots of $\left|\Delta_{S}\right|(2.21)$ for the "ghost brane" solution $\Phi_{a}^{\mathrm{G}}$ at the truncation level $L$. The horizontal axis denotes the value of the parameter $a$.

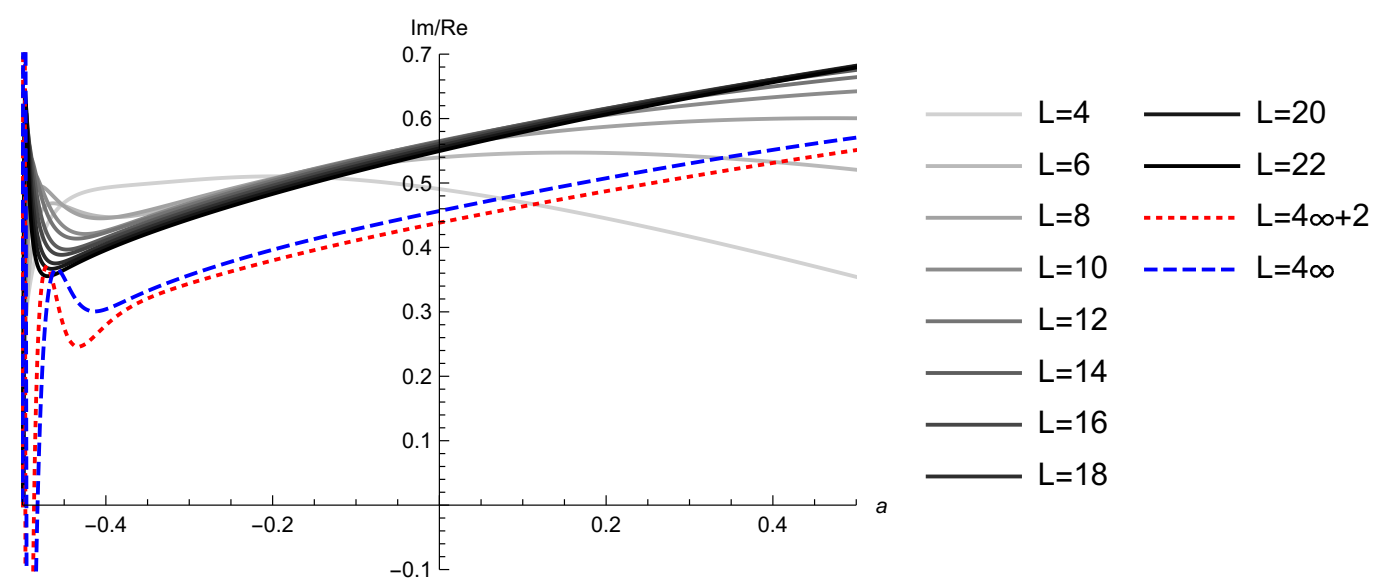

Figure 16. Plots of Im/Re (2.22) for the "ghost brane" solution $\Phi_{a}^{\mathrm{G}}$ at the truncation level $L$. The horizontal axis denotes the value of the parameter $a$.

\section{Concluding remarks}

In this paper, we have numerically constructed the "double brane" solution $\Phi_{a}^{\mathrm{D}}$ and the "ghost brane" solution $\Phi_{a}^{\mathrm{G}}$ in the theory around the TT solution with a real parameter $a$ by using the level truncation method. In particular, in the case $a=0$, they coincide with the "double brane" and "ghost brane" solutions found by Kudrna and Schnabl. In this sense, our solutions are generalization of theirs. We have evaluated the energy $E$ and gauge invariant observable $E_{0}$ for the obtained solutions and calculated $\left|\Delta_{S}\right|$ and $\operatorname{Im} / \operatorname{Re}$ as a consistency check.

From our results, in the large level limit, it seems that $E\left[\Phi_{a}^{\mathrm{D}}\right] \rightarrow E_{2}$ for $a>-1 / 2$ and $\rightarrow E_{2}^{\prime}$ for $a=-1 / 2$, where the real constants, $E_{2}$ and $E_{2}^{\prime}$, satisfy $1<E_{2}<E_{2}^{\prime}$. If $E_{2}=2=E_{2}^{\prime}-1, \Phi_{a}^{\mathrm{D}}$ could be interpreted as double brane solution for $a \geq-1 / 2$ literally, but we observed $E_{2} \sim 1.5$ and $2<E_{2}^{\prime}<3$ from our numerical result. However, the solution $\Phi_{a}^{\mathrm{D}}$ for $a \geq-1 / 2$ is consistent with the interpretation that the TT solution represents the tachyon vacuum at $a=-1 / 2$ and is pure gauge for $a>-1 / 2$ if $E_{2}^{\prime}-E_{2}=1$, which may 
hold. The value of $E_{0}\left[\Phi_{a}^{\mathrm{D}}\right]$ behaves in a similar way to $E\left[\Phi_{a}^{\mathrm{D}}\right]$ numerically. In order to establish the relation $E_{2}^{\prime}-E_{2}=1$, detailed computations around $a=-1 / 2$ for higher levels will be necessary. As for $\Phi_{a}^{\mathrm{G}}$, numerical behavior of the energy and gauge invariant observable is similar to that of $\Phi_{a}^{\mathrm{D}}$, but it is more ambiguous.

We observed that both $\left|\Delta_{S}\left[\Phi_{a}^{\mathrm{D}}\right]\right|$ and $\left|\Delta_{S}\left[\Phi_{a}^{\mathrm{G}}\right]\right|$ approach zero with increasing level, which is consistent with the projected equation of motion: $b_{0} c_{0}\left(Q^{\prime} \Phi+\Phi * \Phi\right)=0$ up to the lowest level. We should check coefficients of higher level states in order to confirm the BRST invariance of solutions in Siegel gauge. It remains as a future work.

The numerical solutions $\Phi_{a}^{\mathrm{D}}$ and $\Phi_{a}^{\mathrm{G}}$ are constructed from complex solutions at level 2 and 4, respectively. Namely, they do not satisfy the reality condition of string field and therefore $E$ and $E_{0}$ for them have imaginary part in general. However, we observed that $\operatorname{Im} / \operatorname{Re}\left[\Phi_{a}^{\mathrm{D}}\right] \rightarrow 0$ with increasing level for $a \geq-1 / 2$. In particular, we found a region of $a$ where $\operatorname{Im} / \operatorname{Re}\left[\Phi_{a}^{\mathrm{D}}\right]=0$ at the level 20 and 22 . In this sense, $\Phi_{a}^{\mathrm{D}}$ is expected to be real at the large level limit for $a \geq-1 / 2$. As for $\Phi_{a}^{\mathrm{G}}$, it seems that $\operatorname{Im} / \operatorname{Re}\left[\Phi_{a}^{\mathrm{G}}\right]$ does not approach zero with increasing level for $a \gtrsim-0.4$, although we found that at $a=-0.5$ and $a=-0.499$, $\operatorname{Im} / \operatorname{Re}\left[\Phi_{a}^{\mathrm{G}}\right]=0$ for the level $L \geq 6$.

We have constructed numerical solutions by Newton's method, where we have to choose appropriate initial configurations for the iterative algorithm. In this paper, we have adopted a choice explained in section 3: 1 . At the lowest truncation level, which is two for $\Phi_{a}^{\mathrm{D}}$ and four for $\Phi_{a}^{\mathrm{G}}$, we have constructed solutions for various values of $a$ from $\Phi_{a=0}^{\mathrm{D}}$ and $\Phi_{a=0}^{\mathrm{G}}$ by varying the value of $a$ little by little. 2. For each value of $a$, higher level solutions have been constructed level by level. However, we can make another choice of initial configurations: $1^{\prime}$. For $a=0$, we construct higher level solutions level by level. $2^{\prime}$. At each level, solutions for $a \neq 0$ are constructed from that for $a=0$ by varying the value of $a$ little by little. We have observed that there is a possibility of obtaining different solutions by $1^{\prime}-2^{\prime}$ from those by $1-2$, where solutions become real near $a=-1 / 2$. Further detailed investigation is a future problem although the procedure $1^{\prime}-2^{\prime}$ takes more time for calculation.

In this paper, we have adopted a method of extrapolations, which is explained in section 3.1. There are two extrapolation values: one is for the truncation level $L=4 \infty+2$ and the other is for $L=4 \infty$, which are plotted in figures by dotted and dashed lines. The difference of them would correspond to an error of the extrapolation. We should justify the extrapolation method or adopt other methods in order to refine the numerical results.

We have performed calculations in Siegel gauge for simplicity. It is desirable to evaluate gauge invariants in other gauges for "double brane" and "ghost brane" solutions to understand them further. We are preparing computations of them in Asano-Kato gauge [18] as in $[19,20] .^{8}$

\section{Acknowledgments}

This work was supported in part by JSPS KAKENHI Grant Numbers JP20K03933, JP20K03972. The numerical calculations were partly carried out on sushiki and XC40 at YITP in Kyoto University.

\footnotetext{
${ }^{8}$ Some comments can be found on calculations in Schnabl gauge in [21].
} 


\section{A Some numerical data for solutions}

\section{A.1 Numerical data for solutions at $a=-1 / 2$}

We list explicit numerical values of solutions in the theory around the TT solution at $a=-1 / 2$ in tables 1 and 2 . They can be compared with those in [5], which correspond to the case $a=0 .{ }^{9}$

The data in table 1 are $E$ (2.15), $E_{0}(2.17),\left|\Delta_{S}\right|$ (2.21), and $\operatorname{Im} / \operatorname{Re}(2.22)$ for the "double brane" solution $\Phi_{a=-1 / 2}^{\mathrm{D}}$ and they correspond to points at $a=-1 / 2$ in figures $1,2,3,4,5,6,7,8$, and 9 . The data in table 2 are $E$ (2.15), $E_{0}(2.17),\left|\Delta_{S}\right|(2.21)$, and $\operatorname{Im} / \operatorname{Re}(2.22)$ for the "ghost brane" solution $\Phi_{a=-1 / 2}^{\mathrm{G}}$ and they correspond to points at $a=-1 / 2$ in figures $10,11,12,13,14,15$, and 16 .

\begin{tabular}{|r|ll|ll|}
\hline$L$ & $E$ & $E_{0}$ & $\left|\Delta_{S}\right|$ & $\operatorname{Im} / \operatorname{Re}$ \\
\hline 2 & $-0.738519+6.42573 i$ & $2.5906-0.611421 i$ & 2.27577 & 0.414127 \\
4 & $-0.520622-3.02656 i$ & $2.7175-0.100579 i$ & 0.624772 & 0.664631 \\
6 & $-0.810759-1.90909 i$ & $2.62035-0.100411 i$ & 0.781281 & 1.15795 \\
8 & $1.2739-1.27992 i$ & $2.59001-0.122603 i$ & 1.24412 & 1.09292 \\
10 & $0.947834-1.30151 i$ & $2.38848+0.0741147 i$ & 0.667769 & 0.80842 \\
12 & $1.55586-1.07501 i$ & $2.35887+0.0660807 i$ & 0.517581 & 0.57895 \\
14 & $1.51726-0.904625 i$ & $2.2996+0.0707516 i$ & 0.431135 & 0.617605 \\
16 & $1.78862-0.768909 i$ & $2.25904+0.0906131 i$ & 0.339793 & 0.501388 \\
18 & $1.7433-0.660931 i$ & $2.22563+0.0962804 i$ & 0.283798 & 0.531292 \\
20 & $1.89055-0.579279 i$ & $2.21258+0.0902456 i$ & 0.232422 & 0.460356 \\
22 & $1.85117-0.508455 i$ & $2.19168+0.0854377 i$ & 0.195764 & 0.481028 \\
\hline
\end{tabular}

Table 1. $E(2.15), E_{0}(2.17),\left|\Delta_{S}\right|(2.21)$, and $\operatorname{Im} / \operatorname{Re}(2.22)$ for the "double brane" solution $\Phi_{a=-1 / 2}^{\mathrm{D}}$ at the truncation level $L$.

\begin{tabular}{|r|ll|ll|}
\hline$L$ & $E$ & $E_{0}$ & $\left|\Delta_{S}\right|$ & $\mathrm{Im} / \mathrm{Re}$ \\
\hline 4 & $-58.3862-1.66004 i$ & $-2.95681+0.472707 i$ & 4.66418 & 0.189811 \\
6 & -24.413 & -2.19698 & 1.91163 & 0 \\
8 & -15.6787 & -1.81939 & 1.29319 & 0 \\
10 & -11.1078 & -1.58933 & 0.87768 & 0 \\
12 & -8.76291 & -1.46405 & 0.65827 & 0 \\
14 & -7.20365 & -1.37387 & 0.503632 & 0 \\
16 & -6.19244 & -1.29508 & 0.40301 & 0 \\
18 & -5.43759 & -1.23428 & 0.327863 & 0 \\
20 & -4.88756 & -1.19106 & 0.273432 & 0 \\
22 & -4.44884 & -1.15705 & 0.230936 & 0 \\
\hline
\end{tabular}

Table 2. $E(2.15), E_{0}(2.17),\left|\Delta_{S}\right|(2.21)$, and $\operatorname{Im} / \operatorname{Re}(2.22)$ for the "ghost brane" solution $\Phi_{a=-1 / 2}^{\mathrm{G}}$ at the truncation level $L . \Phi_{a=-1 / 2}^{\mathrm{G}}$ satisfies the reality condition for $L \geq 6$.

\footnotetext{
${ }^{9}$ Our definition of $\mathrm{Im} /$ Re seems to be different from that in [5]. We suspect that a choice of a basis (or its normalization) is different.
} 


\section{A.2 Coefficients of lowest level states for the solutions}

We list numerical data of three component fields for the solutions, $\Phi_{a}^{\mathrm{D}}$ and $\Phi_{a}^{\mathrm{G}}$, at $a=-1 / 2$ in tables 3 and 4 . They can be compared with those of the solutions at $a=0$ listed in [5]. Furthermore, we plot the coefficient of the lowest level state $c_{1}|0\rangle$, or the tachyon field as a component, of $\Phi_{a}^{\mathrm{D}}$ and $\Phi_{a}^{\mathrm{G}}$ for $a \geq-1 / 2$ in figures $17,18,19$, and 20 . The data in the column of $c_{1}|0\rangle$ in table 3 correspond to points at $a=-1 / 2$ in figures 17 and 18 . The data in the column of $c_{1}|0\rangle$ in table 4 correspond to points at $a=-1 / 2$ in figures 19 and 20 .

Roughly, from figure 18, we can see that the imaginary part of the tachyon field of $\Phi_{a}^{\mathrm{D}}$ vanishes with increasing level for $a \geq-1 / 2$. On the other hand, from figure 20, it seems that the imaginary part of the tachyon field of $\Phi_{a}^{\mathrm{G}}$ remains nonzero with increasing level for $a \gtrsim-0.4$.

\begin{tabular}{|r|lll|}
\hline$L$ & $c_{1}|0\rangle$ & $L_{-2}^{\mathrm{mat}} c_{1}|0\rangle$ & $L_{-2}^{\mathrm{gh} \prime} c_{1}|0\rangle$ \\
\hline 2 & $-1.62421-0.556839 i$ & $-0.144175-0.362514 i$ & $0.179079-0.141459 i$ \\
4 & $-0.811826-0.492565 i$ & $0.0805148-0.211769 i$ & $0.0936975-0.0673882 i$ \\
6 & $-0.617433-0.708114 i$ & $0.169208-0.223221 i$ & $0.0939303-0.0907553 i$ \\
8 & $-0.46957-0.539068 i$ & $0.190683-0.158602 i$ & $0.127765-0.0634097 i$ \\
10 & $-0.594608-0.464836 i$ & $0.110538-0.147441 i$ & $0.0882257-0.0634936 i$ \\
12 & $-0.603815-0.328377 i$ & $0.0657693-0.115888 i$ & $0.084529-0.0536261 i$ \\
14 & $-0.614146-0.359062 i$ & $0.0612609-0.120976 i$ & $0.0742589-0.05115 i$ \\
16 & $-0.598281-0.280436 i$ & $0.0377076-0.0991098 i$ & $0.0693785-0.0454664 i$ \\
18 & $-0.611011-0.304459 i$ & $0.0339221-0.106696 i$ & $0.0626943-0.045099 i$ \\
20 & $-0.589014-0.25289 i$ & $0.0208091-0.0900697 i$ & $0.058484-0.0407456 i$ \\
22 & $-0.601353-0.269899 i$ & $0.0173314-0.0970247 i$ & $0.0539424-0.0413202 i$ \\
\hline
\end{tabular}

Table 3. Coefficients of three lowest level states for the "double brane" solution $\Phi_{a=-1 / 2}^{\mathrm{D}}$ at the truncation level $L$.

\begin{tabular}{|r|lll|}
\hline$L$ & $c_{1}|0\rangle$ & $L_{-2}^{\mathrm{mat}} c_{1}|0\rangle$ & $L_{-2}^{\mathrm{gh} /} c_{1}|0\rangle$ \\
\hline 4 & $-0.761439-0.415565 i$ & $-0.0430207-0.0388406 i$ & $1.44204+0.0481819 i$ \\
6 & -0.360559 & -0.0188028 & 0.675305 \\
8 & -0.238314 & -0.0162807 & 0.440174 \\
10 & -0.152385 & -0.0120697 & 0.310303 \\
12 & -0.11406 & -0.0102722 & 0.234504 \\
14 & -0.0876269 & -0.00885158 & 0.184856 \\
16 & -0.0708067 & -0.00777904 & 0.150024 \\
18 & -0.0584666 & -0.00695981 & 0.124815 \\
20 & -0.0494588 & -0.00625865 & 0.105564 \\
22 & -0.0425024 & -0.00570499 & 0.0907437 \\
\hline
\end{tabular}

Table 4. Coefficients of three lowest level states for the "ghost brane" solution $\Phi_{a=-1 / 2}^{\mathrm{G}}$ at the truncation level $L$. 


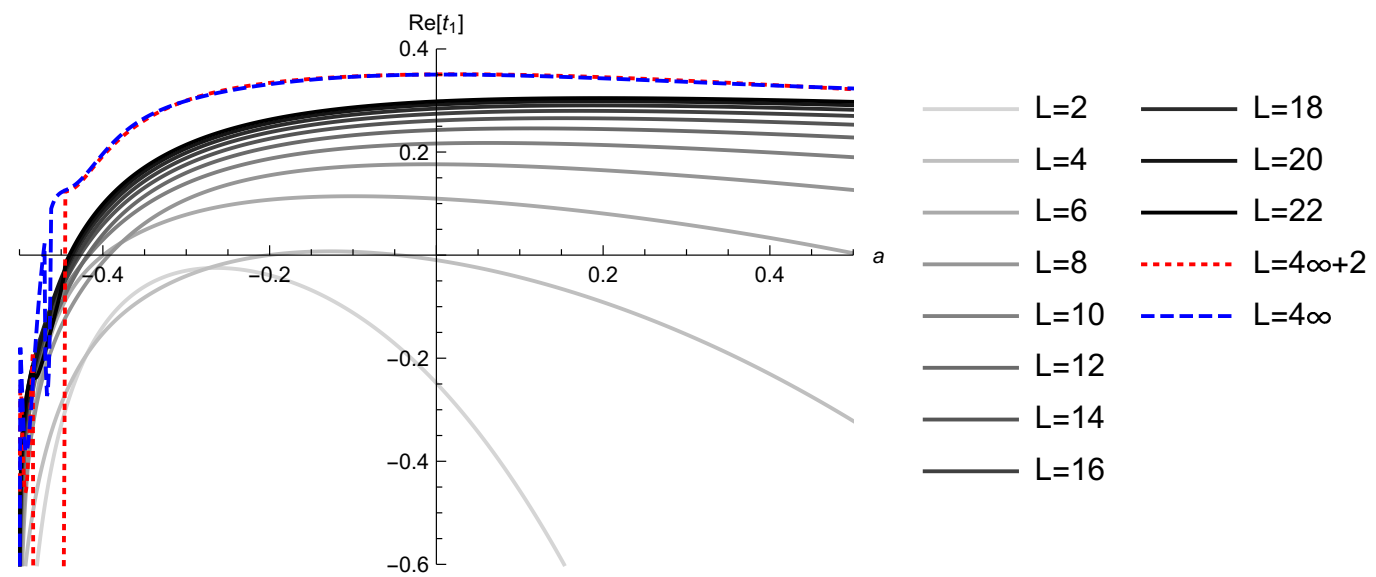

Figure 17. Plots of the real part of the coefficient of the lowest level state $c_{1}|0\rangle$ for the "double brane" solution $\Phi_{a}^{\mathrm{D}}$ at the truncation level $L$. The dotted and dashed lines are extrapolations to $L=4 k+2(k \rightarrow \infty)$ and $L=4 k(k \rightarrow \infty)$, respectively. The horizontal axis denotes the values of the parameter $a$.

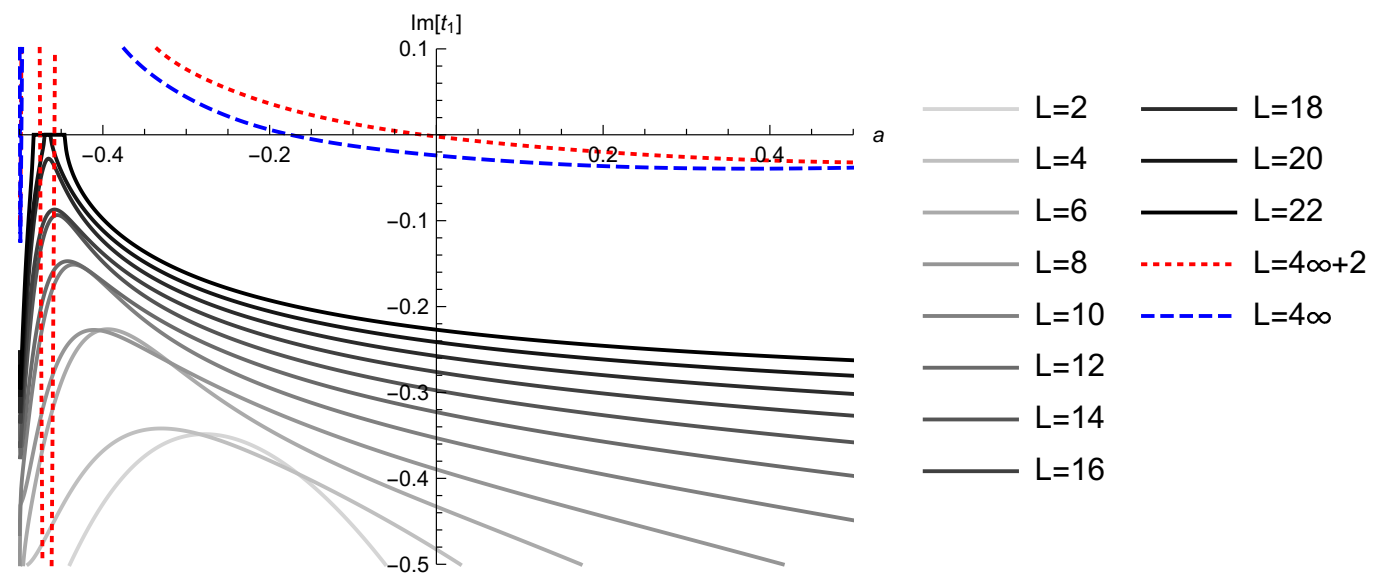

Figure 18. Plots of the imaginary part of the coefficient of the lowest level state $c_{1}|0\rangle$ for the "double brane" solution $\Phi_{a}^{\mathrm{D}}$ at the truncation level $L$. The dotted and dashed lines are extrapolations to $L=4 k+2(k \rightarrow \infty)$ and $L=4 k(k \rightarrow \infty)$, respectively. The horizontal axis denotes the values of the parameter $a$. 


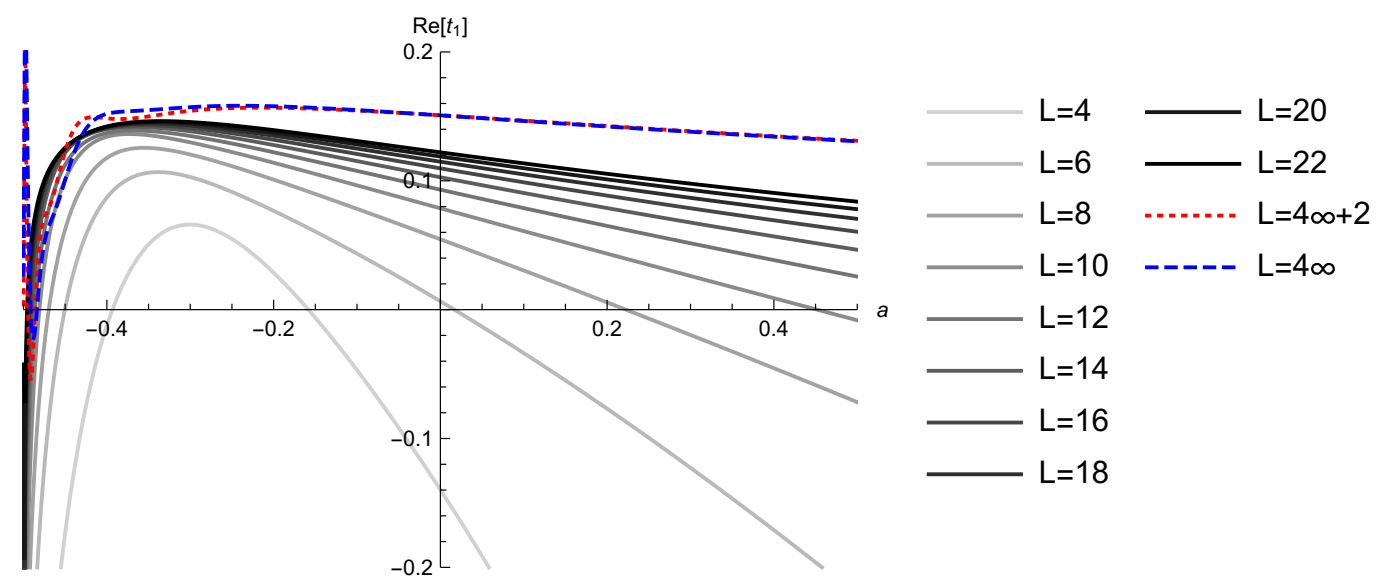

Figure 19. Plots of the real part of the coefficient of the lowest level state $c_{1}|0\rangle$ for the "ghost brane" solution $\Phi_{a}^{\mathrm{G}}$ at the truncation level $L$. The dotted and dashed lines are extrapolations to $L=4 k+2(k \rightarrow \infty)$ and $L=4 k(k \rightarrow \infty)$, respectively. The horizontal axis denotes the values of the parameter $a$.

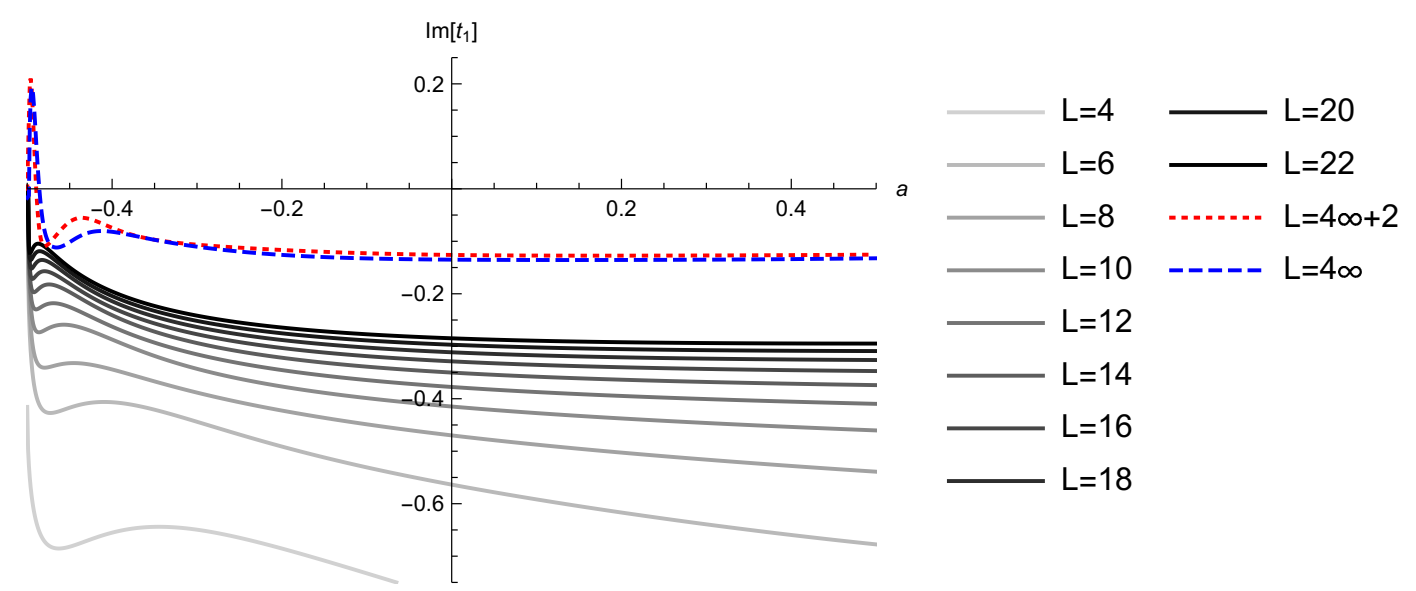

Figure 20. Plots of the imaginary part of the coefficient of the lowest level state $c_{1}|0\rangle$ for the "ghost brane" solution $\Phi_{a}^{\mathrm{G}}$ at the truncation level $L$. The dotted and dashed lines are extrapolations to $L=4 k+2(k \rightarrow \infty)$ and $L=4 k(k \rightarrow \infty)$, respectively. The horizontal axis denotes the values of the parameter $a$. 


\section{A.3 Evaluation of quadratic identities}

It is known that solutions to the equation of motion in open string field theory satisfy certain quadratic identities [22]. Here, we show some numerical data for evaluation of them as a consistency check of numerical solutions in the theory around the TT solution with the parameter $a$. We can derive quadratic identities for solutions to (2.4):

$$
\begin{aligned}
& \left\langle\Phi,\left[Q^{\prime}, L_{n}^{\text {mat }}\right] \Phi\right\rangle=-\frac{65}{54}(-1)^{\frac{n}{2}} n \delta_{n: \text { even }}\left\langle\Phi, Q^{\prime} \Phi\right\rangle, \\
& \left\langle\Phi,\left[Q^{\prime}, L_{n}^{\prime \text { tot }}\right] \Phi\right\rangle=-(-1)^{\frac{n}{2}} n \delta_{n: \operatorname{even}}\left\langle\Phi, Q^{\prime} \Phi\right\rangle,
\end{aligned}
$$

where $L_{n}^{\text {tot }}=L_{n}^{\text {mat }}+L_{n}^{\prime \text { gh }}$, using symmetry of the interaction term of the action (2.1). Particularly, in the case of Siegel gauge solution $\Phi$, if $\left\langle\Phi, c_{0} L(a) \Phi\right\rangle \neq 0$, the ratios of the left hand side and the right hand side of the above equations with $n=2 m$ :

$$
\begin{gathered}
R_{m}= \begin{cases}\frac{\left\langle\Phi, c_{0}\left((1+a) L_{2 m}^{\mathrm{mat}}+a \frac{m-1}{2 m} L_{2 m+2}^{\mathrm{mat}}+a \frac{m+1}{2 m} L_{2 m-2}^{\mathrm{mat}}\right) \Phi\right\rangle}{(-1)^{m} \frac{65}{54}\left\langle\Phi, c_{0}\left((1+a)\left(L_{0}^{\prime \text { tot }}-1\right)+a L_{2}^{\text {tot }}+4 a Z(a)\right) \Phi\right\rangle} & (m=2,3,4, \cdots) \\
\frac{-\left\langle\Phi, c_{0}\left((1+a) L_{2}^{\mathrm{mat}}+a L_{0}^{\mathrm{mat}}+\frac{13}{4} a\right) \Phi\right\rangle}{\frac{65}{54}\left\langle\Phi, c_{0}\left((1+a)\left(L_{0}^{\prime \text { tot }}-1\right)+a L_{2}^{\prime \text { tot }}+4 a Z(a)\right) \Phi\right\rangle} & (m=1)\end{cases} \\
\tilde{R}_{m}= \begin{cases}\frac{\left\langle\Phi, c_{0}\left((1+a) L_{2 m}^{\prime \text { tot }}+a \frac{m-1}{2 m} L_{2 m+2}^{\text {tot }}+a \frac{m+1}{2 m} L_{2 m-2}^{\text {tot }}\right) \Phi\right\rangle}{(-1)^{m}\left\langle\Phi, c_{0}\left((1+a)\left(L_{0}^{\prime \text { tot }}-1\right)+a L_{2}^{\prime \text { tot }}+4 a Z(a)\right) \Phi\right\rangle} & (m=2,3,4 \cdots) \\
\frac{-\left\langle\Phi, c_{0}\left((1+a) L_{2}^{\prime \text { tot }}+a\left(L_{0}^{\prime \text { tot }}+3\right)\right) \Phi\right\rangle}{\left\langle\Phi, c_{0}\left((1+a)\left(L_{0}^{\prime \text { tot }}-1\right)+a L_{2}^{\prime \text { tot }}+4 a Z(a)\right) \Phi\right\rangle} & (m=1)\end{cases}
\end{gathered}
$$

should be one identically. ${ }^{10}$ We note that $\tilde{R}_{1}$ is one at $a=-1 / 2$ trivially.

We list some numerical data for the evaluations of $R_{m}$ (A.3) and $\tilde{R}_{m}$ (A.4) of the "double brane" solution at $a=-1 / 2$ in tables 5 and 6 and those of the "ghost brane" solution at $a=-1 / 2$ in tables 7 and 8 . We also plot the value of $R_{1}$ of $\Phi_{a}^{\mathrm{D}}$ in figures 21 and 22 and that of $\Phi_{a}^{\mathrm{G}}$ in figures 23 and 24. Roughly, from these figures, it seems that $R_{1}$ for $\Phi_{a}^{\mathrm{D}}$ and $\Phi_{a}^{\mathrm{G}}$ approaches one with increasing level although it is unstable around $a=-1 / 2$.

The above numerical behavior is consistent with quadratic identities $R_{m}=1$ and $\tilde{R}_{m}=1$ for solutions at $L=\infty$.

\footnotetext{
${ }^{10}$ The details can be found in [23], where numerical data for the tachyon vacuum and single brane solutions at $a=-1 / 2$, based on the solutions in [4], are listed up to level 26 .
} 


\begin{tabular}{|r|llll|}
\hline$L$ & $R_{1}$ & $R_{2}$ & $R_{3}$ & $R_{4}$ \\
\hline 2 & $0.911488-0.152245 i$ & $-1.27584+0.412182 i$ & 0 & 0 \\
4 & $0.885585+0.021908 i$ & $-1.28084-0.320084 i$ & $-1.2104-0.84751 i$ & 0 \\
6 & $0.8729+0.0201808 i$ & $-1.60019+1.10353 i$ & $-1.59739+1.49291 i$ & $-0.961762+0.978238 i$ \\
8 & $0.950585+0.00241884 i$ & $2.06239+0.84862 i$ & $3.66403-2.82962 i$ & $-2.57524-3.66336 i$ \\
10 & $0.893514+0.0530564 i$ & $-0.248418+1.30193 i$ & $0.9719+1.85136 i$ & $-1.11547+2.07613 i$ \\
12 & $0.937224+0.0359425 i$ & $0.461938+0.777509 i$ & $1.56888+0.298369 i$ & $-0.28761+0.782399 i$ \\
14 & $0.929671+0.058921 i$ & $0.597104+0.95585 i$ & $1.36524+0.815189 i$ & $-0.0304565+1.67951 i$ \\
16 & $0.953995+0.036186 i$ & $0.765202+0.59013 i$ & $1.27848+0.184195 i$ & $0.324349+0.7959 i$ \\
18 & $0.953387+0.0515589 i$ & $0.878275+0.620112 i$ & $1.2123+0.34281 i$ & $0.606532+1.09488 i$ \\
20 & $0.96477+0.0330054 i$ & $0.890854+0.427151 i$ & $1.12715+0.112292 i$ & $0.678698+0.623308 i$ \\
22 & $0.966273+0.0431989 i$ & $0.959863+0.420163 i$ & $1.10173+0.175657 i$ & $0.858197+0.707533 i$ \\
\hline
\end{tabular}

Table 5. $R_{m}(\mathrm{~A} .3)$ of $\Phi_{a=-1 / 2}^{\mathrm{D}}$ at the truncation level $L$ for $m=1,2,3,4$.

\begin{tabular}{|r|llll|}
\hline$L$ & $\tilde{R}_{1}$ & $\tilde{R}_{2}$ & $\tilde{R}_{3}$ & $\tilde{R}_{4}$ \\
\hline 2 & 1 & $-1.49458+0.555977 i$ & 0 & 0 \\
4 & 1 & $-1.3959-0.372793 i$ & $-1.32557-0.977179 i$ & 0 \\
6 & 1 & $-1.82477+1.15122 i$ & $-1.89735+1.67941 i$ & $-1.1746+1.14418 i$ \\
8 & 1 & $2.26372+0.934943 i$ & $4.01471-3.30933 i$ & $-2.90247-4.17631 i$ \\
10 & 1 & $-0.344922+1.40266 i$ & $1.06346+2.07209 i$ & $-1.32026+2.17119 i$ \\
12 & 1 & $0.467894+0.820665 i$ & $1.71676+0.257096 i$ & $-0.355223+0.778747 i$ \\
14 & 1 & $0.596248+1.02744 i$ & $1.5074+0.860021 i$ & $-0.143872+1.76552 i$ \\
16 & 1 & $0.786884+0.614174 i$ & $1.36667+0.142577 i$ & $0.294999+0.813055 i$ \\
18 & 1 & $0.901552+0.656254 i$ & $1.30334+0.320084 i$ & $0.573704+1.15352 i$ \\
20 & 1 & $0.914962+0.437798 i$ & $1.18301+0.0740143 i$ & $0.676277+0.637951 i$ \\
22 & 1 & $0.9852+0.436868 i$ & $1.15838+0.139992 i$ & $0.859524+0.740684 i$ \\
\hline
\end{tabular}

Table 6. $\tilde{R}_{m}$ (A.4) of $\Phi_{a=-1 / 2}^{\mathrm{D}}$ at the truncation level $L$ for $m=1,2,3,4$.

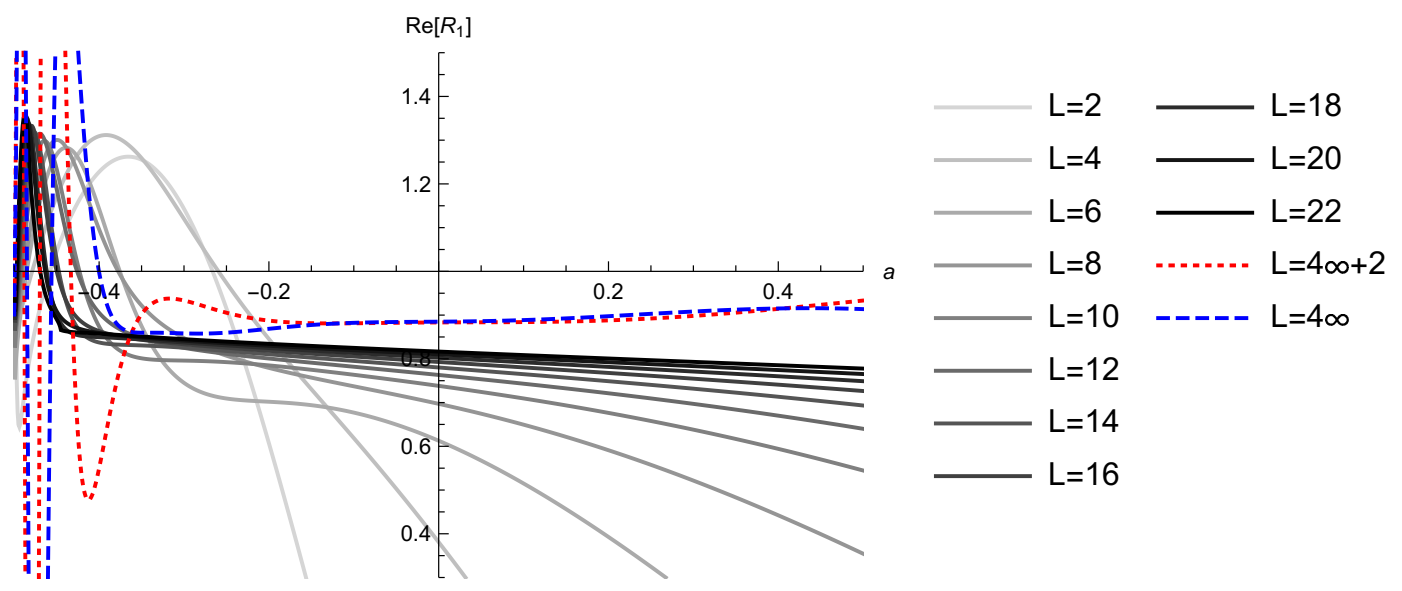

Figure 21. Plots of the real part of $R_{1}$ (A.3) of $\Phi_{a}^{\mathrm{D}}$ at the truncation level $L$. The dotted and dashed lines are extrapolations to $L=4 k+2$ and $L=4 k(k \rightarrow \infty)$, respectively. The horizontal axis denotes the value of the parameter $a$ at $\operatorname{Re} R_{1}=1$. 

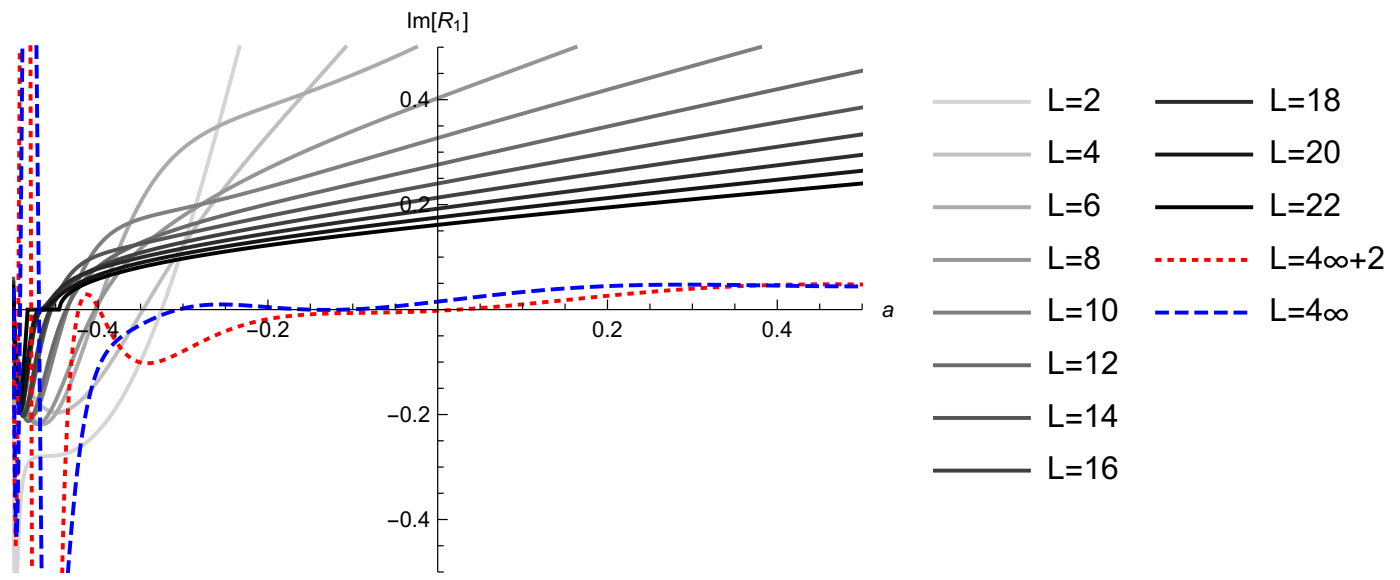

Figure 22. Plots of the imaginary part of $R_{1}$ (A.3) of $\Phi_{a}^{\mathrm{D}}$ at the truncation level $L$. The dotted and dashed lines are extrapolations to $L=4 k+2(k \rightarrow \infty)$ and $L=4 k(k \rightarrow \infty)$, respectively. The horizontal axis denotes the value of the parameter $a$.

\begin{tabular}{|r|llll|}
\hline$L$ & $R_{1}$ & $R_{2}$ & $R_{3}$ & $R_{4}$ \\
\hline 4 & $0.473669-0.0224943 i$ & $0.0290573-0.0136117 i$ & $-0.00391726-0.0214949 i$ & 0 \\
6 & 0.574357 & 0.142781 & 0.0199572 & -0.00288513 \\
8 & 0.6554 & 0.340813 & 0.152805 & 0.0118893 \\
10 & 0.698864 & 0.479036 & 0.328676 & 0.100043 \\
12 & 0.736879 & 0.566131 & 0.462773 & 0.244439 \\
14 & 0.761953 & 0.631667 & 0.555784 & 0.373664 \\
16 & 0.78442 & 0.677713 & 0.625193 & 0.473761 \\
18 & 0.800922 & 0.714594 & 0.674924 & 0.551411 \\
20 & 0.815886 & 0.742564 & 0.714176 & 0.610725 \\
22 & 0.827609 & 0.765937 & 0.743846 & 0.657547 \\
\hline
\end{tabular}

Table 7. $R_{m}$ (A.3) of $\Phi_{a=-1 / 2}^{\mathrm{G}}$ at the truncation level $L$ for $m=1,2,3,4$.

\begin{tabular}{|r|llll|}
\hline$L$ & $\tilde{R}_{1}$ & $\tilde{R}_{2}$ & $\tilde{R}_{3}$ & $\tilde{R}_{4}$ \\
\hline 4 & 1 & $-0.0219749+0.000400093 i$ & $-0.000350886-0.0220953 i$ & 0 \\
6 & 1 & -0.0293363 & 0.029805 & 0.00101128 \\
8 & 1 & 0.16492 & 0.215592 & 0.00493668 \\
10 & 1 & 0.316654 & 0.431604 & 0.0656148 \\
12 & 1 & 0.411717 & 0.583257 & 0.197593 \\
14 & 1 & 0.490697 & 0.68693 & 0.318767 \\
16 & 1 & 0.545656 & 0.758987 & 0.413544 \\
18 & 1 & 0.592945 & 0.809749 & 0.489035 \\
20 & 1 & 0.62858 & 0.847047 & 0.546827 \\
22 & 1 & 0.659964 & 0.874591 & 0.593859 \\
\hline
\end{tabular}

Table 8. $\tilde{R}_{m}$ (A.4) of $\Phi_{a=-1 / 2}^{\mathrm{G}}$ at the truncation level $L$ for $m=1,2,3,4$. 


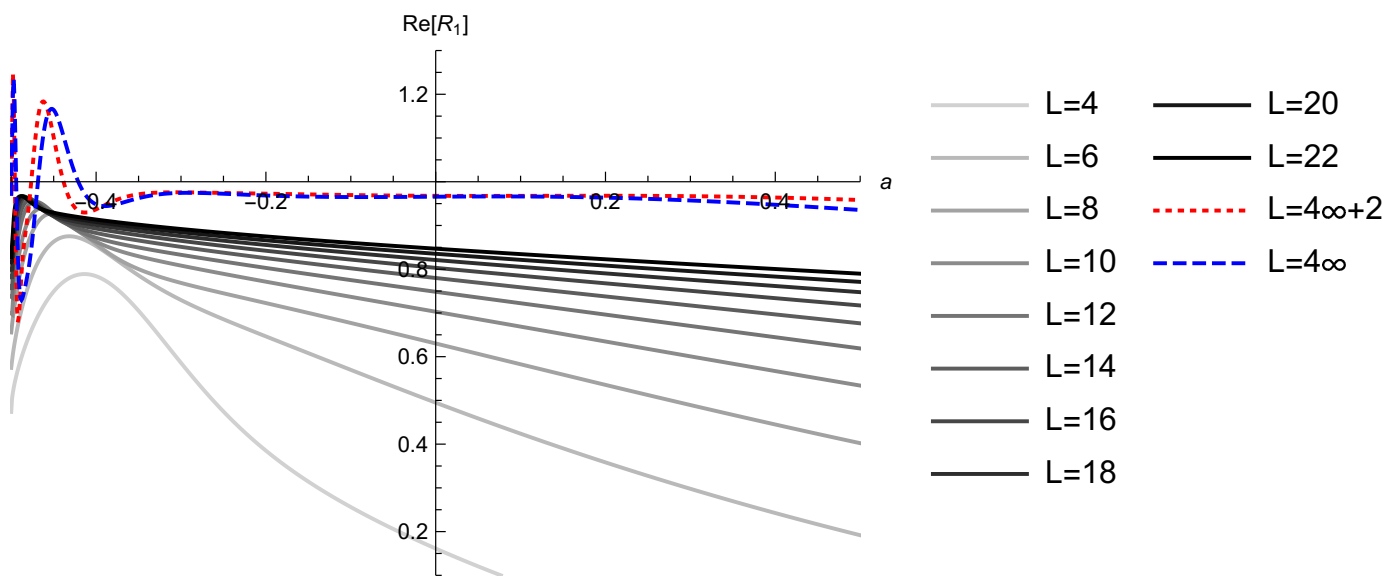

Figure 23. Plots of the real part of $R_{1}$ (A.3) of $\Phi_{a}^{\mathrm{G}}$ at the truncation level $L$. The dotted and dashed lines are extrapolations to $L=4 k+2(k \rightarrow \infty)$ and $L=4 k(k \rightarrow \infty)$, respectively. The horizontal axis denotes the value of the parameter $a$ at $\operatorname{Re} R_{1}=1$.
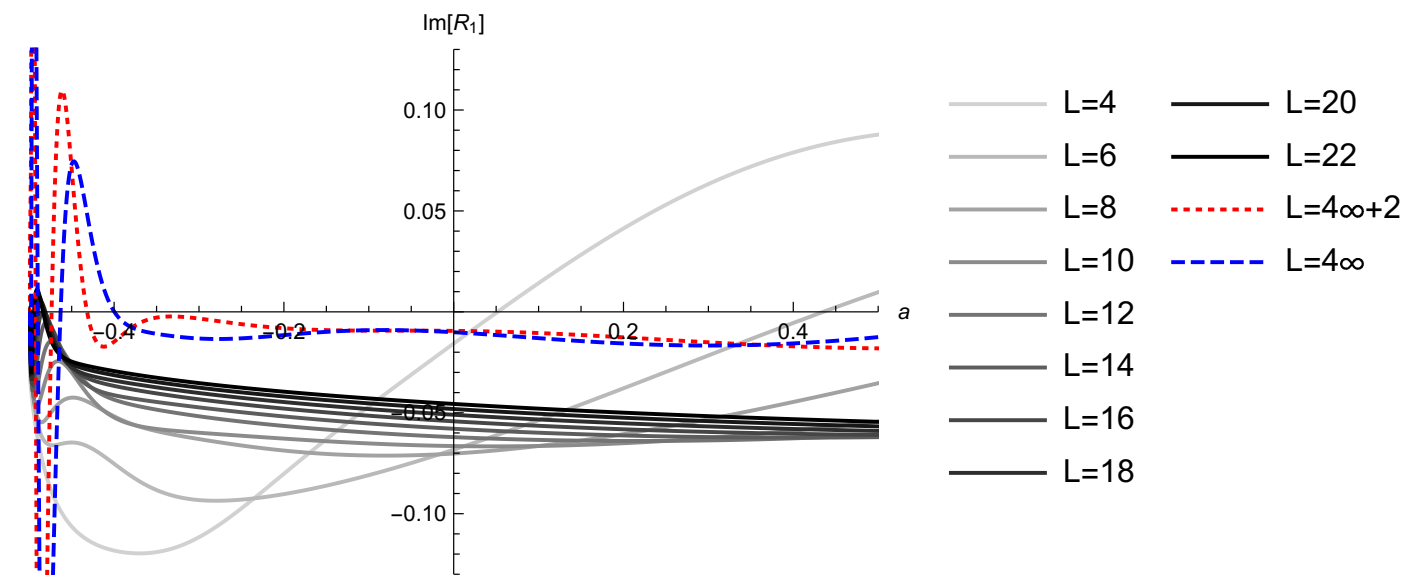

Figure 24. Plots of the imaginary part of $R_{1}$ (A.3) of $\Phi_{a}^{\mathrm{G}}$ at the truncation level $L$. The dotted and dashed lines are extrapolations to $L=4 k+2(k \rightarrow \infty)$ and $L=4 k(k \rightarrow \infty)$, respectively. The horizontal axis denotes the value of the parameter $a$. 
Open Access. This article is distributed under the terms of the Creative Commons Attribution License (CC-BY 4.0), which permits any use, distribution and reproduction in any medium, provided the original author(s) and source are credited.

\section{References}

[1] A. Sen and B. Zwiebach, Tachyon condensation in string field theory, JHEP 03 (2000) 002 [hep-th/9912249] [INSPIRE].

[2] N. Moeller and W. Taylor, Level truncation and the tachyon in open bosonic string field theory, Nucl. Phys. B 583 (2000) 105 [hep-th/0002237] [INSPIRE].

[3] D. Gaiotto and L. Rastelli, Experimental string field theory, JHEP 08 (2003) 048 [hep-th/0211012] [INSPIRE].

[4] I. Kishimoto, On numerical solutions in open string field theory, Prog. Theor. Phys. Suppl. 188 (2011) 155 [INSPIRE].

[5] M. Kudrna and M. Schnabl, Universal solutions in open string field theory, arXiv: 1812.03221 [INSPIRE].

[6] T. Takahashi and S. Tanimoto, Marginal and scalar solutions in cubic open string field theory, JHEP 03 (2002) 033 [hep-th/0202133] [INSPIRE].

[7] I. Kishimoto and T. Takahashi, Open string field theory around universal solutions, Prog. Theor. Phys. 108 (2002) 591 [hep-th/0205275] [INSPIRE].

[8] T. Takahashi, Tachyon condensation and universal solutions in string field theory, Nucl. Phys. B 670 (2003) 161 [hep-th/0302182] [INSPIRE].

[9] I. Kishimoto and T. Takahashi, Vacuum structure around identity based solutions, Prog. Theor. Phys. 122 (2009) 385 [arXiv: 0904.1095] [INSPIRE].

[10] I. Kishimoto and T. Takahashi, Exploring vacuum structure around identity-based solutions, Theor. Math. Phys. 163 (2010) 717 [arXiv:0910.3026] [inSPIRE].

[11] N. Ishibashi, Comments on Takahashi-Tanimoto's scalar solution, JHEP 02 (2015) 168 [arXiv: 1408.6319] [INSPIRE].

[12] I. Kishimoto, T. Masuda and T. Takahashi, Observables for identity-based tachyon vacuum solutions, PTEP 2014 (2014) 103B02 [arXiv:1408.6318] [INSPIRE].

[13] T. Kawano, I. Kishimoto and T. Takahashi, Gauge invariant overlaps for classical solutions in open string field theory, Nucl. Phys. B 803 (2008) 135 [arXiv:0804.1541] [InSPIRE].

[14] I. Ellwood, The Closed string tadpole in open string field theory, JHEP 08 (2008) 063 [arXiv: 0804.1131] [INSPIRE].

[15] M. Schnabl, Analytic solution for tachyon condensation in open string field theory, Adv. Theor. Math. Phys. 10 (2006) 433 [hep-th/0511286] [INSPIRE].

[16] H. Hata and S. Shinohara, BRST invariance of the nonperturbative vacuum in bosonic open string field theory, JHEP 09 (2000) 035 [hep-th/0009105] [INSPIRE].

[17] T. Baba and N. Ishibashi, Energy from the gauge invariant observables, JHEP 04 (2013) 050 [arXiv: 1208.6206] [INSPIRE].

[18] M. Asano and M. Kato, New covariant gauges in string field theory, Prog. Theor. Phys. 117 (2007) 569 [hep-th/0611189] [inSPIRE]. 
[19] I. Kishimoto and T. Takahashi, Numerical evaluation of gauge invariants for a-gauge solutions in open string field theory, Prog. Theor. Phys. 121 (2009) 695 [arXiv:0902.0445] [INSPIRE].

[20] I. Kishimoto and T. Takahashi, Numerical evaluation of gauge invariants for a-gauge solutions in open string field theory, Theor. Math. Phys. 163 (2010) 710 [arXiv:0910.3025] [INSPIRE].

[21] E. Aldo Arroyo and M. Kudrna, Numerical solution for tachyon vacuum in the Schnabl gauge, JHEP 02 (2020) 065 [arXiv: 1908.05330] [INSPIRE].

[22] M. Schnabl, Constraints on the tachyon condensate from anomalous symmetries, Phys. Lett. B 504 (2001) 61 [hep-th/0011238] [INSPIRE].

[23] I. Kishimoto, Numerical evaluation of quadratic identities for classical solutions in open string field theory (in Japanese), to be published in Studies in Liberal Arts and Sciences, Tokyo University of Science. 